\title{
Deployment of an aerial platform system for rapid restoration of communications links after a disaster: a machine learning approach
}

\author{
Faris A. Almalki ${ }^{1} \cdot$ Marios C. Angelides $^{2}$ (D \\ Received: 21 July 2019 / Accepted: 5 October 2019 / Published online: 21 November 2019 \\ (c) The Author(s) 2019
}

\begin{abstract}
Having reliable telecommunication systems in the immediate aftermath of a catastrophic event makes a huge difference in the combined effort by local authorities, local fire and police departments, and rescue teams to save lives. This paper proposes a physical model that links base stations that are still operational with aerial platforms and then uses a machine learning framework to evolve ground-to-air propagation model for such an ad hoc network. Such a physical model is quick and easy to deploy and the underlying air-to-ground (ATG) propagation models are both resilient and scalable and may use a wide range of link budget, grade of service (GoS), and quality of service (QoS) parameters to optimise their performance and in turn the effectiveness of the physical model. The prediction results of a simulated deployment of such a physical model and the evolved propagation model in an ad hoc network offers much promise in restoring communication links during emergency relief operations.
\end{abstract}

Keywords Aerial platforms · Wireless communications · Machine learning

Mathematics Subject Classification 94

Marios C. Angelides

marios.angelides@brunel.ac.uk

Faris A. Almalki

m.faris@tu.edu.sa

1 Department of Computer Engineering, College of Computers and Information Technology, Taif University, Al Hawiyah, Kingdom of Saudi Arabia

2 Department of Electronic and Computer Engineering, College of Engineering, Design and Physical Sciences, Brunel University London, Uxbridge UB8 3PH, UK 


\section{Introduction}

During man-made or natural disaster situations, wireless communication systems have the additional merit that they are less vulnerable to physical damage. When disaster strikes, terrestrial communication links are often disrupted, yet for disaster relief workers, such links are essential during rescue operations. The importance of establishing reliable communications in the aftermath of a natural disaster was clearly highlighted in 2017 in Florida during one of the most damaging Hurricanes in American history with winds reaching up to $220 \mathrm{~km} / \mathrm{h}$ [1]. According to the Federal Communications Commission (FCC), 80 people have died, more than $65 \%$ of Florida's residents were left without electricity, more than $50 \%$ of cell sites went out of service either caused by the damage and/or backup power battery depleting and backup generators running out of fuel. Approximately 893,409 from the 1,115,752 subscribers in the affected areas in Florida lost all connections. In response, more than 7000 emergency cellular equipment such as portable towers have been used on the first days to establish Ad hoc connectivity [2]. It took the local authority in Florida about 180 days to restore and reconnect 4G Long-Term Evolution (LTE) services to cover the entire state [1], [3]. Furthermore, the limited coverage range of terrestrial towers may not justify the effort required to restore one or more terrestrial towers in the aftermath of a disaster. Under such conditions, it is essential to have a large-scale and robust communication network for broadcasting instructions to people, seeking rescue aid, as well as providing inter-departmental communications. Disruptions caused by physical damage can be incredibly costly and time consuming to restore, as they require maintenance or sometimes replacement of complex network hardware to re-establish communications. This is especially problematic if major installations such as terrestrial towers or fibre-optic cables are involved [4-7].

Mobility, expandability, affordability, connectivity, productivity, surveillance and disaster management for public safety and security are catalysts in outer space communication systems, whether aerial platforms or satellites [8]. The authors of [4, 5] and [9] offer several examples of space communication systems that had successfully help establish ad hoc networks during disaster relief operations. This is mostly based on outer space communication systems covering a wide area, offering deployment flexibility, forecasting disaster evolution, providing last-mile connectivity, re-configurability, and in case of emergency and disaster relief operations, offering unique Line of Sight (LoS) advantage [10]. However, satellite systems are not without limitations, for instance, propagation delay, handover complexity, high power consumption and cost. All these in addition to environmental issues such as high gas emissions during satellite launches, as well as signals that have no regard for geographical or political boundaries, which might or might not be a desirable feature $[5,6,11]$.

Aerial platforms, on the other hand, are of flexible size since they are usually helium-filled and solar powered airships and can be used for various applications and services such as telecommunications, broadcasting, surveillance, emergency services, and navigation. Their position in the sky could take advantage of the strengths of terrestrial and satellite communication systems, whilst avoiding some of their weaknesses. Deploying an Ad hoc network using aerial platforms for disaster relief swiftly bridges communication gaps through a soft infrastructure, a fast start-up time, grad- 
ual growing, on-demand capacity assignment, with low capital investment, as well as low ongoing operating costs $[4,6,11]$. Delving deeper, the low cost and speed of deployment of aerial platforms in comparison to that of satellites may quickly restore connectivity for the hundreds of thousands of victims and users in the disaster zones that lost their connection and without high energy consumption. Furthermore, the use of aerial platforms may help with restoring communications selectively; for example, replacing both overloaded and broken terrestrial links, or around hospitals and emergency rooms or in areas where rescue teams are operating.

Considering aerial platforms in emergency situations with an optimized propagation model is scarcely reported in the literature and where it is the constituent models do not utilise the full range parameters to optimise its performance let alone taking into consideration QoS and GoS parameters. This paper proposes a physical model that uses aerial platforms for re-establishing connectivity in the immediate aftermath of a natural disaster and then uses a machine learning framework to evolve an ATG propagation model at different altitudes. The evolved propagation model considers optimal altitudes and elevation angles of the constituent aerial platforms to achieve connectivity with assured QoS and GoS for rescue teams in a typical urban and dense population environment. Such optimization would help in meeting both the demand and nature of an unplanned event.

The rest of this paper is organized as follows: Section II reviews related works on aerial platforms technology for emergency response operations. Section III presents our proposed physical model of aerial platforms alongside a non-optimized ATG propagation model. Section IV presents our proposed machine learning framework that uses RBFNN to evolve an optimised ATG propagation model. Section V simulates the physical model and discusses the predicted performance results. Section VI presents a proof-of-concept deployment and section VII concludes.

\section{Related research review}

Aerial platforms are increasingly seen as an innovative solution to the last-mile problem. They offer many of the satellite advantages, but without the distance penalty. Receivers may experience a better signal quality, as the system offers LoS communications, hence, less propagation delay in relation to satellite systems. Our review of relevant literature reveals that there has been some consideration of the capabilities of aerial platforms for emergency situations. This section reviews issues that reportedly affect the performance of aerial platforms as an alternative to a Base Station: propagation models, altitude and elevation angles and performance indicators.

\subsection{Propagation models}

Propagation models play a vital role in wireless communications since they measure the power density of an electromagnetic wave as it propagates over space from which several useful information can be obtained such as path loss (PL) and coverage, which in turn are important for monitoring system performance and network planning [10, 
12]. This subsection discusses the various types of propagation models introduced in the literature for emergency situations. Researchers in [12] have used three stochastic geometry propagation models, i.e. Single Knefe Edge, Deygout, and Bullington, in the evaluation of aerial platform performance to support mobile receivers to broadcast alerts in emergency situations. The results of these models show reasonable results when aerial platforms reach up to an altitude of $100 \mathrm{~m}$. However, their recommendation is to consider propagation models that serve higher altitudes and seek lower PL along with higher Received Signal Strength (RSS). In their future work, they recommend consideration of buildings as 3D obstacles in urban scenarios.

In [13] an optimal aerial platform location is examined for emergency conditions to minimize PL and maximize RSS. An ATG propagation model is considered at an altitude of $1 \mathrm{~km}, 2 \mathrm{GHz}$ frequency band and transmission power set at $40 \mathrm{dBm}$. Their results show that the maximum urban radius achieved is $20 \mathrm{~km}$ with PL and RSS of $130 \mathrm{~dB}$ and $82 \mathrm{dBm}$ respectively. Authors in [14] highlight the behaviour of an LTE network in a disaster scenario using an aerial platform at $17 \mathrm{~km}$ altitude. The propagation model considered is ATG, transmission power is set at $30 \mathrm{dBm}$, and multibeam antenna gain at $38.7 \mathrm{dBi}$. The simulation results show that the RSS floats between $-70 \mathrm{dBm}$ and $-110 \mathrm{dBm}$, and the system can restore $92 \%$ of throughput. Nevertheless, they conclude that further aerial platform configurations and link designs are required to enhance QoS results. In [15] the authors discuss the energy efficiency of emergency response with wireless communications using hybrid aerial platforms and mobile terrestrial networks. A Rician propagation model, whose type is not sufficiently accurate, is used to analyse the effectiveness of a cooperative relay strategy. The simulation results indicate that the channel quality of cooperative communication strategies perform better than direct communication links thus yielding better energy efficiency. Their future work aims at studying the mobility effects on cooperative relaying strategies with multiple aerials relay platforms. However, their model does not consider the aerial platform's altitude, PL, RSS, and coverage range.

In [16] researchers investigate the performance of various propagation models, i.e. Two-ray, WinnerII, and Free-space Pathloss (FSPL), for emergency scenarios before selecting the best fitting model. Their result show that different parameters such as attenuation, altitude, and frequency of operation, and flying altitude, have effect on the pathloss model. Additionally, their results infer that the use of the empirical Winner II model with some modification in correction factors would be a useful model in emergency communication systems for disaster scenarios. This highlights the importance of seeking optimization. The authors in [17] highlight several open research issues for aerial platforms one of which is channel modelling. It is considered, in their view, as the most distinctive characteristic communication for all wireless communication including aerial platform systems. Thus, it is vital to optimize the channel model to achieve feasible outcomes not only in terms of link budget parameters, and QoS, but also in terms of energy saving, which remains a key challenge for UAV communications.

Authors in [18] investigate the performance of LTE and Wireless Fidelity (Wi-Fi) technologies in an urban Australian emergency scenario using a tethered low aerial platform. A ray tracing ATG path loss model and three empirical propagation models are simulated at many aerial platform altitudes, and four network performance indicators are measured, i.e. PL, outage probability, delay, and throughput. Their 
results show that LTE outperforms WiFi under all conditions, while it is inferred that cost, coverage, and deployment time should be considered for suitable selection of technology for low aerial platforms. One important enhancement offered by aerial platforms is their ability to increase the footprint area compared to terrestrial networks due to an increased LoS probability. However, this depends on altitude, frequency band, and antenna specifications, which have to be carefully considered.

\subsection{Altitude and elevation angle}

A trail of Emergency Broadband Access Network (EBAN) is designed in [19] to provide wide area hotspots for emergency relief in Indonesia using a tethered-blimp balloon. The evaluation is based on measured Received Signal Level (RSL) and Signal-to-Noise Ratio (SNR). In this trial, WiFi and Worldwide Interoperability for Microwave Access (WiMAX) services are considered. At an altitude of $0.4 \mathrm{~km}$ above ground, the balloon's coverage area is $47.39 \mathrm{~km}^{2}$ with a constant $54 \mathrm{Mb} / \mathrm{s}$ downlink throughput, but as coverage increases to $72 \mathrm{~km}^{2}$ throughput fluctuates. In [20] authors highlighted the importance of providing high QoS for mobile users with an effective coverage using aerial platforms. They argue that the optimal altitude of aerial platforms that may achieve a wide footprint coverage against energy efficiency is an open research challenge and so is the elevation angle that may yield more LoS connectivity.

Researchers in [21] investigate the performance of 4G LTE, and WiFi multimode base stations installed on aerial stations to deliver coverage for first responders in emergency situations. Directional antennas are utilized in the aerial platform to provide either macrocell, microcell or picocell coverage. Their results show that the performance of different link segments, whether LTE or WiFi, at varying aerial-station altitudes between 0.5 and $2 \mathrm{~km}$ is quite high. However, packet delay increases as the number of parallel services rises. In [16] researchers investigate the performance of various propagation models for emergency scenario before selecting the best fitting model. Their results show that the parameters of attenuation, altitude, frequency of operation, and flying altitude effect the pathloss model. Their results suggest that the altitude of an aerial platform may lead to a trade-off between better coverage at higher altitudes and better signal quality at lower altitudes. Thus, finding an optimal altitude that may achieve better performance is vital.

In [22] the authors study the coverage of an aerial platform system equipped with $\mathrm{WiFi}$ access points to provide terrestrial users with wireless communication services for short-term events and/or emergency situations. Their results show that at an altitude of $0.5 \mathrm{~km}, 2.4 \mathrm{GHz}$ frequency band and transmission power set at $35 \mathrm{dBm}$, the maximum urban radius achieved is $6 \mathrm{~km}$, with a PL of $120.5 \mathrm{~dB}$ and an RSS of $-80 \mathrm{dBm}$. The authors consider the use of an aerial platform at an altitude of $0.3 \mathrm{~km}$ using LTE to enable communications for emergency or temporary events. At such an altitude, RF signals can overcome most ground-level obstacles, which enables most users to enjoy a LoS connectivity to the aerial platform. One limitation that arises when increasing the aerial platform's altitude to increase coverage range, is an increase in power con- 
sumption. Authors in [23] investigate the performance optimization of drones used for public safety and contributing towards IoT and smart cities. One of their research findings focuses on the ratio between LoS probability and elevation angle in a function of an aerial platform altitude, where LoS probability increases as elevation angle increases at a fixed altitude. The elevation angle varies between $30^{\circ}$ and $70^{\circ}$ in urban and dense urban areas. However, changing the altitude and elevation angle seems to be based on trial and error.

Researchers in [24] design an aerial platform with a Wi-Fi prototype system to provide wireless services to emergency areas. Whilst a Wi-Fi signal is transmitted for a few tens of meters, with the support of an aerial platform transmission power and altitude this can be extended to reach up to $25 \mathrm{~km}$ using directional antennas. Results establish the feasibility of first respond communications using a flexible aerial platform. The authors in [25] exploit the ABSOLUTE project which relates to emergency $4 \mathrm{G}$ communications to aid disaster traffic management using a tethered aerial platform. In their experiment an aerial platform is inflated and launched at a $300 \mathrm{~m}$ altitude within $10 \mathrm{~min}$. At such an altitude, a $72 \mathrm{~km}$ LoS is achieved. Thereafter, devices with batteries within the footprint coverage are immediately connected. The results suggest that aerial platforms offer advantages over a terrestrial tower not only in terms of coverage but also in terms of data rate, due to the LoS connectivity. Their work highlights two remaining challenges that relate to energy consumption, and antenna types. The authors of [26] and [27] present several examples involving aerial platforms and portable base stations. [26] Presents a tethered aerial platform prototype in Indonesia at an altitude of $0.66 \mathrm{~km}$ above ground that is deployed in populated areas in the aftermath of natural disasters to support communications between medical relief units. Its coverage ranges up to $78.54 \mathrm{~km}^{2}$, with a downlink throughput of up to $54 \mathrm{Mbps}$ on $5.8 \mathrm{GHz}$ WiFi.

\subsection{Performance indicators}

This subsection discusses issues that may affect the performance of aerial platforms such as frequency band, antenna specifications, interference, delay, and power consumption. In aerial platform technology, both WiMAX and LTE perform well and are suggested as good candidates for better coverage, whether in LoS or nLoS, increased capacity and less interference [13, 28-30]. The researchers in [31] present two scenarios of disaster monitoring and disaster relief using aerial platforms. First, during the tsunami in Japan, aerial platforms are deployed to cover a $100 \mathrm{~km}$ diameter in a mega cellular layout. Second, during a forest fire in Cyprus, aerial platforms are deployed to cover a $60 \mathrm{~km}$ diameter in a multi cellular layout. Authors in [32] investigate the challenges of WiMAX, WiFi, LTE, ZigBee, and XBee wireless technologies for enabling aerial drone platform in Alpine environments, in order to support short term winter events and provide a novel but viable solution in emergency and rescue situations in a hostile environment. The candidacy of an omni-directional antenna type is considered in this case and WiMAX has been suggested as a suitable wireless technology for drone communications for several reasons: flexibility, safety, QoS, interference, throughput, installation, and coverage area. The WiFi gives a satisfactory performance for Internet 
access and easily achieves a LoS for rural users but with challenging consequences for urban users. WiMAX offers better capacity, less interference, and has better coverage with no LoS (nLoS) where an object block signals.

Recommendations for future work includes an investigation of the impact of emergency traffic on the various communication interfaces at different altitudes [28, 33]. A set of aerial platforms are considered in [34] for emergency communication using delay-tolerant networks (DTN) on sub-GHz frequencies. Their aim is to use these as decision support aerial nodes to enhance sharing vital information including Maps, videos, and other operational information between rescue teams, victims, inter-departmental, and headquarters. The use of DTN shows the usefulness of such deployment in an environment that lacks continuous network connectivity. The experiment results show that flying aerial platforms at $120 \mathrm{~m}$ above ground using a low frequency of $760 \mathrm{MHz}$ enables detecting victims buried $1 \mathrm{~m}$ deep underground. However, delay seems to be an issue. In [35], the authors present a survey on heterogenous wireless networks of satellites, aerial platforms, and terrestrial stations in emergency situations. The authors discuss the key technologies of these systems, and most importantly highlight three major challenges: security, interoperability and QoS, i.e. bandwidth, delay, and loss.

The authors of [5] and [36] argue that ad hoc networks with aerial platforms are deployable quickly with an average deployment time of approximately $10 \mathrm{~min}$ and a coverage range of approximately a $25 \mathrm{~km}$ radius. In contrast, Vodafone's Instant Networks [37], a portable terrestrial base station, may be deployed in approximately 40 min of reaching the deployment site and may yield a coverage range of approximately a $5 \mathrm{~km}$ radius. The authors of [38] consider a Contingency Cellular Network $(\mathrm{CCN})$ in China that re-connects base stations with wireless links and portable power generators. Their results show that it requires the cooperation of approximately 14 teams to set up the CNN. Authors in [39] present an algorithm that calculates the optimal placement to cover an area using different station types, i.e. portable terrestrial stations (PTSs), and aerial platforms. Both assume being equipped with LTE technology for random situations. The authors present a case from the disaster caused by Hurricane Katrina. Their results confirm the advantage of deploying aerial platforms in terms of high bandwidth utilization, wide coverage, and required number of base stations to cover a specified area in relation PTSs. However, increasing the number of aerial platforms may cause interference with terrestrial stations. Therefore, it is recommended to positioning aerial platforms on the boundaries of the disaster area, so that interference is reduced whilst leaving no coverage gaps. Considering multiple antenna technology improves performance as indicated by Alamouti's scheme Multiple-Input Multiple-Output (MIMO) antenna technology. The scheme maximises capacity and improves QoS and coverage extension range.

The effect of MIMO antennas on near space solar powered platforms performance and capacity is discussed in [40-42], where it is argued that the antenna gain is optimized, to prevent users from experiencing weak radio across many miles. The ITU's International Mobile Telecommunications-Advanced (IMT-Advanced) standard for $4 \mathrm{G}$ offers access to various telecommunication services and supports mobile applications for various environments that offer high data rates to users. Power consumption at the receiver end has been considered widely in assessing the performance of wire- 
less sensor networks (WSN) and several approaches report improvements in QoS and power consumption. In [43] a Bit Error Rate (BER) power scheduling scheme in WSN is proposed to avoid retransmitting data. Simulation results show that the total energy consumption is reduced. Researchers in [44] highlight a direct communication link design between WSN and space-based communications, where it is inferred that MIMO antennas could improve results.

In [45] the authors cover performance analysis on WSN integration between aerial platforms and satellites. Results indicate that an aerial platform's wireless sensors are preferred as their shortest PL compares favourably to satellites, and their LoS connectivity compares favourably to terrestrial systems, which contributes to reducing BER and improving power consumption from ground sensors. The authors in [42] present a low cost and off-the-shelf balloon trial in Slovenia at altitudes ranging between 0.6 and $1.1 \mathrm{~km}$ above ground. Its coverage reaches up to a $9 \mathrm{~km}$ radius and its throughput varies depending on modulation and frequency band. Their RSS performance is improved by controlling the beam direction, thus taking advantage of MIMO diversity gain. In [46] the authors design an aerial platform for enabling communications and information sharing among the first responders and citizens during disaster recovery operations. Based on the experiment, their proposed model shows reasonable results in terms of coverage and link budget parameters. However, the aerial platform altitude is considered low at $30 \mathrm{~m}$ but yields low transmission power and antenna gain and in turn LoS connectivity.

The authors in [47] introduce a four-tier hybrid ad hoc architecture for post-disaster communication that aims to measure latency and assess network resource utilization using a customized ONE Simulator. The extensive simulation results show the effectiveness of the proposed architecture of resource utilization within a range of $8.5 \mathrm{~km}^{2}$. Furthermore, the packet delivery probability degrades gradually with an increase in traffic load. The study does not offer any stats about link budget parameters or altitude. In [48] the authors propose an optimization approach based on stochastic geometry and a mathematical framework to achieve better coverage probability for multiple aerial platforms in post-disaster sites. The results show that the average achievable rate was strongly affected by the aerial platforms' altitudes and transmit powers. Energy efficiency and channel muddling are presented as future work for emergency communications via aerial platforms.

Table 1 summarises our findings from our review of the literature in terms of the issues addressed and possible gaps that remain and have motivated our own research work.

\section{A physical and non-optimised propagation models using aerial platforms}

Our related work review in the previous section has considered reported aspects of aerial platforms for short-term emergency communications. Considering aerial platforms in any emergency situations with an optimized propagation model is scarcely reported in the literature, let alone considering QoS and GoS. This section aims at first to introduce our physical model for re-establishing communications with aerial 
Table 1 Related research windup

\begin{tabular}{|c|c|c|}
\hline Issue & Addressed & Gap \\
\hline Propagation models & $\begin{array}{l}\text { ATG model extended, Empirical } \\
\text { models adapted for ad hoc planning } \\
{[12,16]}\end{array}$ & $\begin{array}{l}\text { Empirical models' limited scope [17, } \\
18]\end{array}$ \\
\hline Platform altitude & Variable $[19,21]$ & $\begin{array}{l}\text { Low altitude limits coverage, } \mathrm{LoS} \\
\text { connectivity, high shadowing effect } \\
{[16,21,23]}\end{array}$ \\
\hline Elevation angle & $\begin{array}{l}\text { Widespread calculation in ATG model } \\
\text { to obtain wide footprint, Achieving } \\
\text { better connectivity at low elevation } \\
\text { angles with directive antenna [13, } \\
18]\end{array}$ & $\begin{array}{l}\text { Some elevation angles are unsuitable } \\
\text { for all environments, Selecting an } \\
\text { optimal elevation angle during } \\
\text { emergencies is open issue }[20,23]\end{array}$ \\
\hline Link budget & Parameter set expanded [17] & $\begin{array}{l}\text { Parameter set expanded further, } \\
\text { Enhancing power consumption } \\
\text { through minimizing PL, Exploiting } \\
\text { altitude and RSS trade-off }[9,12,15]\end{array}$ \\
\hline Antenna type & $\begin{array}{l}\text { Smart, directive: coverage footprint } \\
\text { calculation }[14,18,25]\end{array}$ & $\begin{array}{l}\text { MIMO limited consideration for } \\
\text { diversity gain }[38,44]\end{array}$ \\
\hline QoS & $\begin{array}{l}\text { Various techniques to minimize power } \\
\text { consumption }[17,32,35]\end{array}$ & $\begin{array}{l}\text { Trade-off between pathloss and power } \\
\text { consumption to guarantee long } \\
\text { lifespan connection for WSN [35, } \\
45]\end{array}$ \\
\hline GoS & - & $\begin{array}{l}\text { Lack of consideration for aerial } \\
\text { platforms }\end{array}$ \\
\hline
\end{tabular}

platforms in the immediate aftermath of a natural or man-made disaster and then set up an initial, non-optimized, ATG propagation model.

\subsection{A physical model for re-establishing connectivity using aerial platforms}

Figure 1 illustrates through the use of the 3D Remcom Wireless InSite software tool our physical model in action.

Several aerial platforms deployed at different altitudes and elevation angles to support the disaster relief operations of six rescue teams in an urban environment. These rescue teams are distributed randomly across a city of $17 \mathrm{~km}$ footprint radius to cover different scenarios for both LoS and NLoS conditions accrued. The figure shows a modern and developed city, where neighbourhoods are paved, and population distribution is equal. It also shows the physical 3D architecture of the re-established wireless communications with two main segments: a space segment with aerial platforms, and a ground segment. The ground stations (those that are still operational) are linked using both backhaul links and host gateways to external networks, and intermediate wireless sensor nodes on the ground. Such an ad hoc network may still contain several remote sensors that collect ground segment data. WSNs have been widely adopted in numerous disaster management and emergency response cases, such as disaster relief, gas leakage, and water level monitoring, all of which can help 


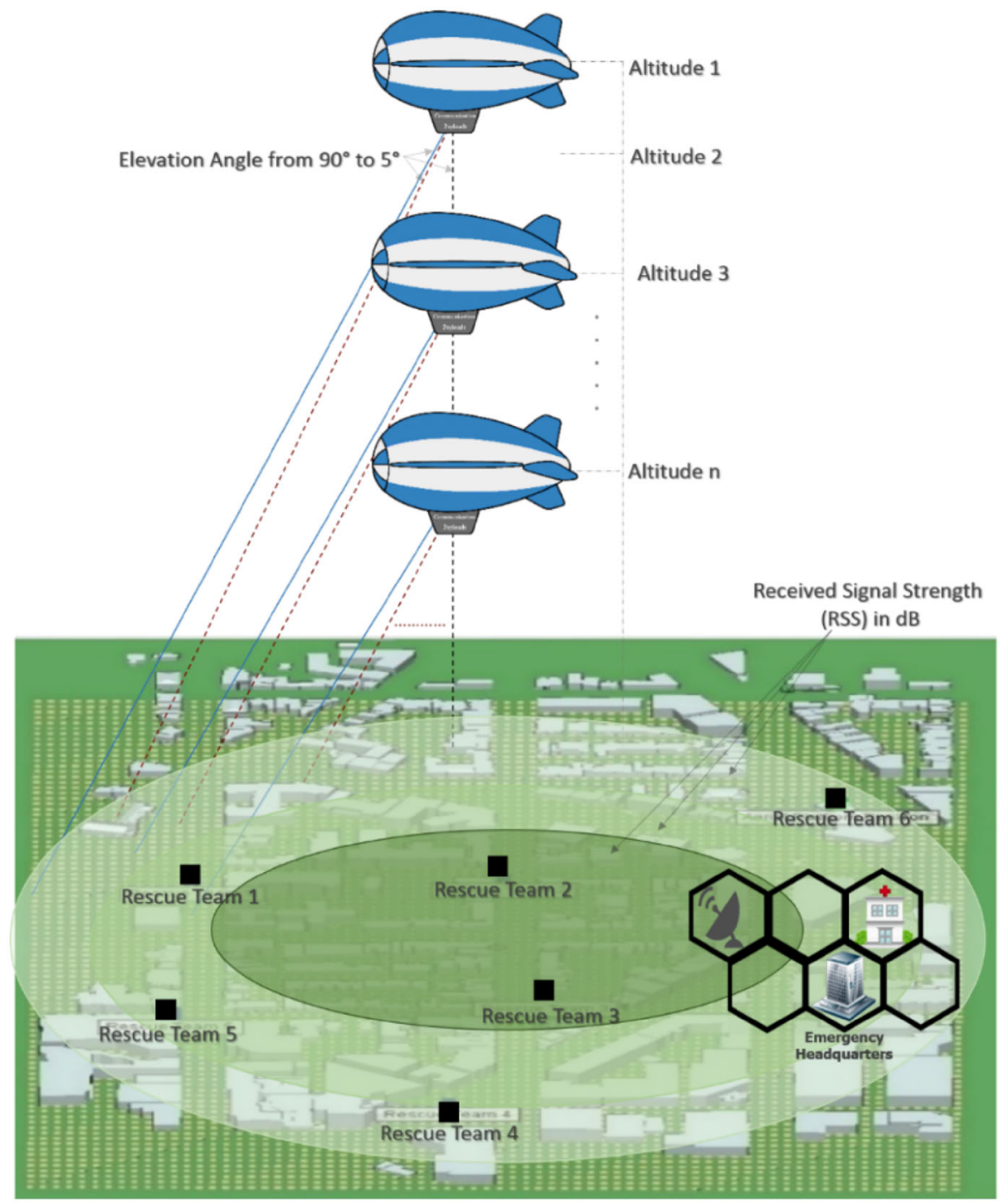

Fig. 1 3D network architecture for emergency situations

towards Public Safety (PS). This may lead to an environment which is more stable and secure.

\subsection{Non-optimised propagation model}

Propagation models predict signal attenuation or PL as a measure of the power density of an electromagnetic wave as it propagates through space from a transmitter. Calculating PL helps monitor system performance, network planning and coverage in order to achieve strong RSS. RSS plays a significant role not only in terms of signal strength, throughput and footprint coverage, but also has direct effects on QoS 
and GoS. Many factors may affect a signal when propagating to a maximum distance: transmitter antenna height, receiver antenna height, terrain type, antenna gain, transmission power, LoS connectivity, frequency of operation, and distance between transmitter and receiver. Several competing propagation models have been proposed over the years but whilst they collectively raise many shortcomings such as limited altitude up to few tens of meters, lack of cover across different environments and low perdition accuracy, they also exhibit several advantages.

We have developed our initial non-optimized, propagation model based on the ATG model because it exhibits advantages in relation to high altitude, wide coverage range, adaptation across different terrains [49-52]. The ATG model utilises a fuller range of link budget parameters alongside the MIMO diversity gain in order to enhance LoS connectivity, reduce PL and extend coverage range. The ATG channel characteristics along with the link budget parameters can help with monitoring system performance in dense urban environments in order to optimise it to meet the demand and/or nature of the planned urban area for robust and reliable wireless communications during emergencies.

The range of link budget parameters included in our initial, non-optimised, ATG propagation model has been extended and, in addition, QoS and GoS parameters have also been included all of which will be used to predict its performance: Firstly, link budget parameters, i.e. $P L, R S S$, Throughput $(D)$, coverage radius $(R)$; secondly, QoS parameters, i.e. $B E R$ and Energy per Bit to Noise Spectrum Density (Eb/No); thirdly, GoS parameters, i.e. $P_{B}$, and $P_{C}$. Evaluating PL, RSS, D, and R can help in Ad hoc network planning, wide coverage footprint, receivers' LoS, and data rates which may all vary in response to geomorphology characteristics. GoS aims at measuring the ratio of users accessing a trunked system during the busiest hours in the aftermath of an emergency.

\subsubsection{Link budget prediction}

Calculation of total $\mathrm{P}_{\mathrm{L}}$ is as follows:

$$
\mathrm{PL}_{\mathrm{T}}=\rho_{\mathrm{LoS}} \times \mathrm{PL}_{\mathrm{LoS}}+\rho_{\mathrm{NLoS}} \times \mathrm{PL}_{\mathrm{NLoS}}
$$

The probability of having LoS connections at an elevation angle of $\theta$, depending on the type of environment is given by:

$$
\begin{gathered}
\rho_{\mathrm{LoS}}=\mathrm{a}-\frac{\mathrm{a}-\mathrm{b}}{1+\left[\frac{\theta-\mathrm{c}}{\mathrm{d}}\right]^{\mathrm{e}}} \\
\rho_{\mathrm{NLOS}}=1-\mathrm{P}_{\mathrm{LoS}}
\end{gathered}
$$

The path losses for LoS and NLoS are as follows:

$$
\operatorname{PL}_{\mathrm{LoS}}(\mathrm{dB})=20 \log \frac{4 \pi(\mathrm{f})(\mathrm{d})}{\mathrm{c}}+\eta_{\mathrm{LoS}}
$$




$$
\operatorname{PL}_{\mathrm{NLoS}}(\mathrm{dB})=20 \log \frac{4 \pi(\mathrm{f})(\mathrm{d})}{\mathrm{c}}+\eta_{\mathrm{NLoS}}
$$

where $\eta_{\text {LoS }}, \eta_{\text {NLoS }}$ are average additional losses to free space depending on type of environment, whereas a, b, c, d, and e are ITU-R empirical parameters for different elevation angles and environments, as ATG depends on LoS or nLoS to include the shadowing effects [49-52].

The rest of the link budget parameters that are considered are: RSS, throughput, and coverage radius. RSS depends on $P_{t}, P_{L}, G\left(h_{t}\right), G\left(h_{r}\right)$ as well as connector and cable loss L. Throughput D depends on bandwidth B and SNIR using Shannon's formula. The RSS, and D are as follows:

$$
\begin{gathered}
R S S=P_{t}+G\left(h_{t}\right)+G\left(h_{r}\right)-P_{L}-L \\
D=B \times \log (1+S N I R)
\end{gathered}
$$

\subsubsection{QoS prediction}

The link quality between an aerial platform sink and ground sensors relies on factors such as elevation angle between sink and sensors, operation frequency, transmission power, transmitter and receiver antenna gains, RSS, atmospheric conditions, bit rate and link distance $[53,54]$. Thus, the QoS is a measure of the performance of WSNs via two indicators: Eb/No and BER. These two QoS indicators are significant in monitoring energy consumption without resorting to sensor power enhancement or external power sources. This can be achieved by reducing fading, low propagation PL and high RSS. The performance of wireless sensors in this paper can be analysed by considering two main QoS parameters: Eb/No and the BER which highlights the performance of different digital modulation schemes. These parameters are considered in the link budget in order to set QoS guarantees for the applications they serve, and that can be expressed as:

$$
\begin{gathered}
\frac{\mathrm{E}_{\mathrm{b}}}{\mathrm{N}_{0}}=\frac{\mathrm{C}}{\mathrm{N}}+10 \log \mathrm{BW}-10 \log \mathrm{R}_{\mathrm{b}} \\
\frac{\mathrm{C}}{\mathrm{N}}=\mathrm{EIRP}-\mathrm{P}_{\mathrm{L}}-\mathrm{A}_{\mathrm{R}}+\left(\frac{\mathrm{G}}{\mathrm{T}}\right)-10 \log \frac{\mathrm{K} \mathrm{BW}}{0.001} \\
\mathrm{EIRP}=\mathrm{P}_{\mathrm{t}}+\mathrm{G}_{\mathrm{t}}+\mathrm{G}_{\mathrm{r}}-\mathrm{L} \\
\frac{\mathrm{G}}{\mathrm{T}}=\mathrm{G}_{\mathrm{r}}-10 \log \mathrm{T}
\end{gathered}
$$

where $\mathrm{C} / \mathrm{N}$ is carrier power measured in $\mathrm{dB}, \mathrm{BW}$ is bandwidth measured in $\mathrm{MHz}, \mathrm{R}_{\mathrm{b}}$ is the data rate for modulation specific Quadrature Phase Shift Keying (QPSK), the Equivalent Isotopically Radiated Power (EIRP) is measured in $\mathrm{dBm}, \mathrm{P}_{\mathrm{t}}$ is transmitter power, $G_{t}$ is transmitter antenna gains, $G_{r}$ is receiver antenna gains, $L$ is connector and cable loss, $A_{R}$ is rain attenuation and atmospheric gas attenuation which are negligible, 
$\mathrm{K}$ is Boltzmann's constant $\left(1.38065 \times 10^{-23}\right), 0.001$ represents normalization, $\mathrm{G} / \mathrm{T}$ is the ratio of the receiver antenna gain to system noise temperature measured in $\mathrm{dB} 0$, $\mathrm{T}$ is an effective temperature in this model $(310 \mathrm{~K})[53,54]$. The link performance parameter for signal quality is BER/Probability of Error which in turn is directly related to Eb/No. Thus, we calculate the BER as a function of Eb/No for a QPSK modulation in an Additive white Gaussian noise (AWGN) channel as:

$$
\mathrm{BER}=\frac{1}{2} \operatorname{erfc} \sqrt{\frac{\mathrm{E}_{\mathrm{b}}}{\mathrm{N}_{0}}}
$$

\subsubsection{GoS prediction}

The chosen channel model can be also evaluated using the concept of Trunking. A trunked mobile radio system provides access to users on demand from an available number of channels. A small number of channels can accommodate a large random number of users due to limited radio spectrum. GoS aims to measure the ratio of users accessing a trunked system during the busiest hours which in this case are immediately after the natural or man-made disasters. GoS is modelled by considering two performance indicators: firstly, the probability that a call is blocked $\mathrm{P}_{\mathrm{B}}$, and secondly, the probability that a call is delayed by a certain queuing time $\mathrm{P}_{\mathrm{D}}$. These two GoS indicators are significant in monitoring the performance of an aerial platform in relation to frequency reuse with a view of optimising the performance of GoS to increase system capacity and meet the footprint of a disaster area's size and population density $[55,56]$. The probability of being blocking $\mathrm{P}_{\mathrm{B}}$ is expressed as follows:

$$
\begin{aligned}
\mathrm{P}_{\mathrm{B}} & =\frac{\frac{\mathrm{A}^{\mathrm{C}}}{\mathrm{C} !}}{\sum_{\mathrm{i}=0}^{\mathrm{C}} \frac{\mathrm{A}^{\mathrm{i}}}{\mathrm{C} !}} \\
\mathrm{A}_{\mathrm{u}} & =\lambda_{\mathrm{T}} \times \mathrm{H} \\
\mathrm{A} & =\mathrm{U} \times \mathrm{A}_{\mathrm{u}} \\
\mathrm{A}_{\mathrm{c}} & =\mathrm{A} \times \mathrm{R}_{\mathrm{n}}
\end{aligned}
$$

where $\mathrm{P}_{\mathrm{B}}$ is the ratio between the number of lost calls and the total number of calls, $\mathrm{C}$ is a rounding number of channels multiplied by obtaining an RSS for each rescue team, $\mathrm{A}$ is total traffic, $\mathrm{H}$ is the call duration, $\lambda_{\mathrm{T}}$ is arrived calls rate, $\mathrm{U}$ is number of users, $A_{u}$ is the call rate per user, $A_{c}$ is carried traffic, and $R_{n}$ is number of cells. The probability of delay $\mathrm{P}_{\mathrm{D}}$ is expressed as follows:

$$
\begin{aligned}
& \left(P_{D}>0\right)=\frac{A^{C}}{A^{C}+C !\left(1-\frac{A}{C}\right) \sum_{i=0}^{C-1} \frac{A^{i}}{C !}} \\
& \left(P_{D}>t\right)=\left(P_{d}>0\right) \times \exp \left(\frac{-\left(\frac{C-A}{t}\right)}{H}\right)
\end{aligned}
$$




$$
\mathrm{D}=\left(\mathrm{P}_{\mathrm{D}}>0\right) \times \frac{\mathrm{H}}{\mathrm{C}-\mathrm{A}}
$$

where the average delay time in seconds, $\mathrm{D}$, of delayed calls, $\mathrm{P}_{\mathrm{D}}>0$ is the likelihood that a call is initially denied access to a channel in the system, $P_{D}>t$ is the probability that the delayed call is forced to wait more than $t$ seconds.

\section{A machine learning framework for evolving an optimal propagation model}

We propose a machine Learning framework that evolves an optimal propagation model from the initial, non-optimised, propagation model developed in the previous section using the same link budget, QoS, and GoS parameters at different altitudes, elevation angles in order to achieve three main goals with reference to the demand in and, nature of, the planned area: Firstly, increasing wireless connectivity alongside reducing coverage gaps between rescue teams and victims and emergency relief headquarters; secondly, reducing resource energy consumption alongside increasing QoS; thirdly, increasing system capacity alongside increasing GoS. The machine learning framework utilises a Radial Basis Function (RBF) Neural Network (NN) firstly to avoid local minima and secondly as there is good coverage of the input space. We have experimented with a variety of deep NN models in the past and none of these would significantly outperform shallow NN like RBF; they would only add complexity to the computation process with their requirement of multiple layers.

Figure 2 demonstrates our proposed Neural Network Framework and the ensuing decision-making process as a three-stage approach. The size of the input, hidden and output layers has been set at 8, 7 and 1 respectively. A random selected sample from the data set that has been provided by the WiMAX company Airspan [57] is used as input to the NN for training using Stochastic Gradient Descent. The predicted results for PL, RSS, D, R, BER, Eb/No, $\mathrm{P}_{\mathrm{B}}$, and $\mathrm{P}_{\mathrm{C}}$ are used to populate the input vector to the RBFNN. Thus, during the first stage, the link budget parameters, i.e. PL, RSS, D, and R, are used in the evolution of the propagation model are predicted from the non-optimised propagation models and the platform altitudes and elevation angles using the 3D Remcom Wireless InSite software tool and MATLAB. During the second stage, the QoS parameters, i.e. BER and Eb/No, and GoS parameters, i.e. $\mathrm{P}_{\mathrm{B}}$, and $\mathrm{P}_{\mathrm{C}}$, are used in the evolution of the evolved propagation model are predicted from the parameters obtained during the first stage. During the (optimisation) third stage, the parameter predictions obtained during the first and second stages are used as the input vector into a RBFNN [58-62]. The second layer is a hidden layer whereby the neurons are activated by the RBF using Gaussian functions. The third layer produces the network output Y. Whilst the transformation mapping from the input space to the hidden layer space is linear, mapping from input to output is nonlinear. The learning rate is accelerated, and the usual local minimum issue is avoided. These three layers are expressed mathematically as they are shown in Eqs. (20)-(27). 


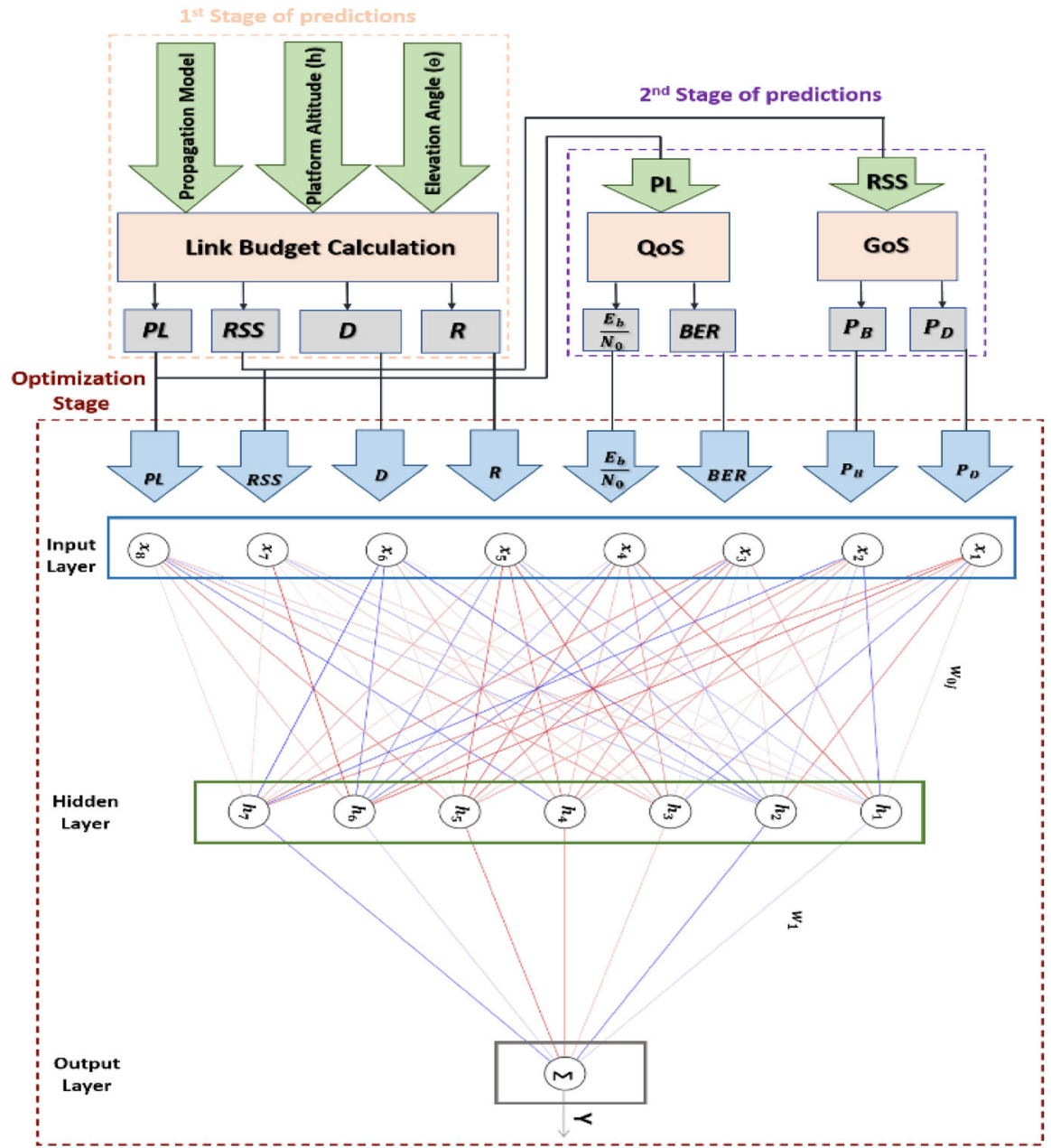

Fig. 2 The proposed NN framework

Equation (20) represents the input vector of the network:

$$
\mathrm{X}=\left[\mathrm{x}_{1}, \mathrm{x}_{2}, \mathrm{x}_{3}, \ldots \mathrm{x}_{\mathrm{n}}\right]^{\mathrm{T}}
$$

where $\mathrm{x}_{1}$ is $\mathrm{PL}, \mathrm{x}_{2}$ is RSS, $\mathrm{x}_{3}$ is $\mathrm{D}, \mathrm{x}_{4}$ is $\mathrm{R}, \mathrm{x}_{5}$ is BER, $\mathrm{x}_{6}$ is Eb/No, $\mathrm{x}_{7}$ is $\mathrm{P}_{\mathrm{B}}$, and $\mathrm{x}_{8}$ is $P_{C}$, and the $X$ values vary according to the change of altitudes and elevation angles of aerial platform.

Equations (21)-(24) represent the hidden layer which holds several nodes and applies a nonlinear transformation to the input variables using the RBF as the Gaussian function:

$$
\mathrm{H}=\left[\mathrm{h}_{1}, \mathrm{~h}_{2}, \mathrm{~h}_{3}, \ldots \mathrm{h}_{\mathrm{n}}\right]^{\mathrm{T}}
$$




$$
\begin{aligned}
\mathrm{h}_{\mathrm{n}} & =\exp \left(-\frac{\left\|\mathrm{X}-\mathrm{C}_{\mathrm{n}}\right\|^{2}}{2 \mathrm{~b}_{\mathrm{n}}^{2}}\right) \\
\mathrm{C} & =\left[\mathrm{C}_{1}, \mathrm{C}_{2}, \mathrm{C}_{3}, \ldots \mathrm{C}_{\mathrm{n}}\right]^{\mathrm{T}} \\
\mathrm{B} & =\left[\mathrm{b}_{1}, \mathrm{~b}_{2}, \mathrm{~b}_{3}, \ldots \mathrm{b}_{\mathrm{n}}\right]^{\mathrm{T}}
\end{aligned}
$$

where $\mathrm{H}$ represents the radial vector of the RBF network, $\mathrm{h}_{\mathrm{n}}$ is a multivariate Gaussian function, $\mathrm{C}$ is the centre vector of the network, and $\mathrm{B}$ is the radial width vector.

Equation (25) represents the weight vector of the network:

$$
\mathrm{W}=\left[\mathrm{w}_{1}, \mathrm{w}_{2}, \mathrm{w}_{3}, \ldots \mathrm{w}_{\mathrm{n}}\right]^{\mathrm{T}}
$$

The learning phase proceeds in the perceptron by changing connection weights after each piece of data is processed. The weights of $\mathrm{W}_{0 \mathrm{j}}$ for every input are initialised as follows: $\mathrm{PL}=0.2, \mathrm{RSS}=0.9, \mathrm{D}=0.2, \mathrm{R}=0.2, \mathrm{BER}=0.5, \mathrm{~Eb} / \mathrm{No}=0.5, \mathrm{P}_{\mathrm{B}}=$ $0.9, \mathrm{P}_{\mathrm{D}}=0.9$. The high weight of RSS, $\mathrm{P}_{\mathrm{B}}$ and $\mathrm{P}_{\mathrm{D}}$, denotes higher priority in order to provide better channel performance during the setup of emergency communications. However, the weights of $\mathrm{W}_{\mathrm{ji}}$ are randomly selected and updated in the perceptron of hidden layer as the learning phase proceeds.

Equation (26) represents the network output as a linearly weighted sum of the number of base functions in the hidden layer:

$$
\mathrm{Y}=\mathrm{F}_{0}\left(\sum_{\mathrm{j}=0}^{\mathrm{N}} \mathrm{W}_{0 \mathrm{j}}\left(\mathrm{F}_{\mathrm{h}}\left(\sum_{\mathrm{i}=0}^{\mathrm{N}} \mathrm{W}_{\mathrm{ji}} \mathrm{h}_{\mathrm{n}}\right)\right)\right)
$$

where $F_{h}$ and $F_{0}$ are the activation functions of the neurons from the hidden layer and output layer, respectively.

Equation (27) customises Eq. (26) with actual parameters:

$$
\begin{aligned}
\mathrm{Y} & =\left(\mathrm{W}_{\mathrm{PL}}+\mathrm{h}_{1} \mathrm{~W}_{1}\right)+\left(\mathrm{W}_{\mathrm{RSS}}+\mathrm{h}_{2} \mathrm{~W}_{2}\right)+\left(\mathrm{W}_{\mathrm{D}}+\mathrm{h}_{3} \mathrm{~W}_{3}\right)+\left(\mathrm{W}_{\mathrm{R}}+\mathrm{h}_{4} \mathrm{~W}_{4}\right) \\
& +\left(\mathrm{W}_{\mathrm{BER}}+\mathrm{h}_{5} \mathrm{~W}_{5}\right)+\left(\mathrm{W}_{\mathrm{Eb} / \mathrm{No}}+\mathrm{h}_{6} \mathrm{~W}_{6}\right)+\left(\mathrm{W}_{\mathrm{P}_{\mathrm{B}}}+\mathrm{h}_{7} \mathrm{~W}_{7}\right)+\left(\mathrm{W}_{\mathrm{P}_{\mathrm{D}}}+\mathrm{h}_{8} \mathrm{~W}_{8}\right)
\end{aligned}
$$

Selecting the most optimal value is done by applying k-means clustering using a Gaussian mixture distribution as shown in Eq. (28) through to (30). This can be done by specifying the beta coefficients and setting sigma to the mean distance between the cluster centre and other points in the cluster. k-means starts by initializing the centre for first pattern of the cluster, which includes the optimized values of different parameters at various aerial platform altitudes and elevation angles. Then RBF neurons compete at every iteration until the $\mathrm{NN}$ reaches either a stage where there are no updated centres or reaches the maximum number of iterations [58-62].

Sigma is calculated as:

$$
\sigma=\frac{1}{\mathrm{~m}} \sum_{\mathrm{i}=1}^{\mathrm{m}}\left\|\mathrm{x}_{\mathrm{i}}-\mu\right\|
$$


where, $\mathrm{m}$ is the number of training samples belonging to this cluster, $\mathrm{x}_{\mathrm{i}}$ is the $\mathrm{ith}$ training sample in the cluster, $\mu$ is the cluster centroid.

$\beta$ can be computed from the obtained $\sigma$ as:

$$
\beta=\frac{1}{2 \sigma^{2}}
$$

The Gaussian data distribution $\mathrm{p}(\mathrm{x})$ is assumed to be a weighted sum of $\mathrm{K}$ number of distributions of the mixture and can be expressed as:

$$
\mathrm{p}(\mathrm{x})=\sum_{\mathrm{i}=1}^{\mathrm{K}} \pi_{\mathrm{k}} \mathcal{N}\left(\mathrm{x} \mid \beta \sum_{\mathrm{i}}\right)
$$

where $\pi_{\mathrm{k}}$ is the proportion of data generated by the $\mathrm{k}$-th distribution, $\mathcal{N}\left(\mathrm{x} \mid \beta \sum_{\mathrm{i}}\right)$ is the multidimensional Gaussian function with mean vector $\beta$ and covariance matrix $\left(\Sigma_{\mathrm{i}}\right)$.

During the process of selecting the best optimized value, the network progresses by adaptively fine-tuning the free parameters of the system based on the corrections which minimize the mean squared error (MSE), between inputs $\mathrm{y}_{\mathrm{i}}$ and the desired output $\mathrm{d}_{\mathrm{i}}$, which represents the pounds of the parameters that are considered to improve channel performance. The MSE is regarded as an evaluation criterion of the proposed NN and can be expressed as:

$$
\operatorname{MSE}=\frac{1}{2} \sum_{j=1}^{N}\left(y_{i}-d_{i}\right)^{2}
$$

Thus Eqs. (28)-(31) represent the Gaussian mixture distribution for optimization before selection of the most optimal value at the bell curve peak. The bell curve is visualised as a multi-dimension shaded mesh plot in MATLAB. Equation (31) is a performance indicator of the NN framework, where a well-trained ANN should have a very low MSE at the end of the training phase.

Once communications links are being restored, network operators experience a much higher than normal volume of data traffic because of the traffic generated by the rescue and relief operations as well as all other non-emergency and residential communications. Thus, the proposed machine learning approach is aimed at offering robust ad hoc network planning, wide coverage footprint, receiver LoS, and low call loss and delay. The flowchart on Fig. 3 illustrates the operations of the proposed RBFNN using Gaussian distribution, MSE and regression analysis. This includes data selection, creating and training a network, and evaluating performance.

\section{Physical model simulation and predicted results analysis}

Literature reports on testing WiMAX for aerial platforms is scarce despite offering the advantage of independent links with minimum interference, especially for short term events and emergency situations. Our simulation serves predictions of a fuller range of 
Fig. 3 Flowchart of the proposed RBFNN

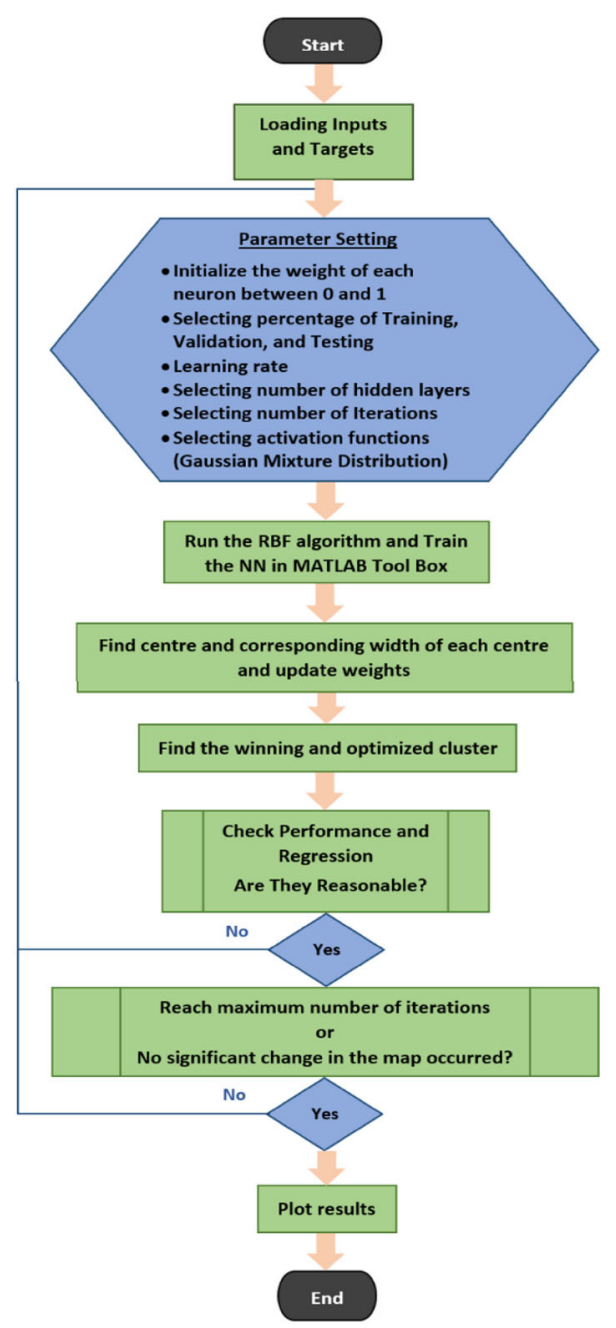

link budget parameters using WiMAX MIMO antenna specifications. Specification of simulation parameters for MIMO antennas have been provided by the Airspan mobile WiMAX Telecom Company [57]. Early stage predictions are obtained by applying Eqs. (1)-(19) in 3D Remcom Wireless InSite software tool and MATLAB. Elevation angles vary between $5^{\circ}$ and $90^{\circ}$ at different altitudes of the Aerial Platform which float between 1 and $20 \mathrm{~km}$ above ground.

On the transmitter side the frequency band is $2.5 \mathrm{GHz}$ with Power $=37 \mathrm{dBm}$, Antenna Gain $=17 \mathrm{dBi}$, Diversity gain $=5 \mathrm{dBi}$, Rx Sensitivity $=-90 \mathrm{dBm}$, and loss $=5 \mathrm{~dB}$. On the Receiver side Power $=27 \mathrm{dBm}$, Antenna Gain $=2 \mathrm{dBi}$, Diversity gain $=2 \mathrm{dBi}$, Rx Sensitivity $=-88 \mathrm{dBm}$, loss $=0.5 \mathrm{~dB}$. The aerial platform altitudes, denoted by transmitter antenna height $h_{t}$, are set at $5 \mathrm{~km}$, and $20 \mathrm{~km}$, respectively 
Table 2 Early stage numerical predictions

\begin{tabular}{|c|c|c|c|c|c|c|}
\hline \multirow[b]{2}{*}{ Parameters } & \multicolumn{6}{|c|}{ Performance of rescue teams for $h_{t}=5 \mathrm{~km}$ of $\theta=15^{\circ}$} \\
\hline & Team 1 & Team 2 & Team 3 & Team 4 & Team 5 & Team 6 \\
\hline PL (dB) & -128.52 & -129.06 & -129.01 & -131.81 & -133.00 & -130.46 \\
\hline RSS (dBm) & -57.3 & -58.15 & -58.13 & -60.43 & -63.81 & -59.72 \\
\hline $\mathrm{D}(\mathrm{Mb} / \mathrm{S})$ & 2.34 & 2.33 & 2.33 & 2.21 & 2.20 & 2.24 \\
\hline $\mathbf{R}(\mathbf{k m})$ & 12.50 & 12.35 & 12.50 & 10.64 & 10.50 & 11.50 \\
\hline$P_{B}$ & 0.42 & 0.44 & 0.46 & 0.53 & 0.57 & 0.49 \\
\hline$P_{D}$ & 0.35 & 0.36 & 0.38 & 0.46 & 0.49 & 0.42 \\
\hline BER & $1 \times 10^{-6}$ & $1 \times 10^{-6}$ & $1 \times 10^{-6}$ & $1 \times 10^{-6}$ & $1 \times 10^{-6}$ & $1 \times 10^{-6}$ \\
\hline$\frac{\mathrm{E}_{\mathrm{b}}}{\mathrm{N}_{0}}(\mathrm{~dB})$ & 18.50 & 18.85 & 18.79 & 20.57 & 22.69 & 19.34 \\
\hline \multicolumn{7}{|c|}{ Performance of rescue teams for $h_{t}=20 \mathrm{~km}$ of $\theta=30^{\circ}$} \\
\hline PL (dB) & -129.00 & -129.13 & -129.62 & -132.41 & -133.5 & -131.00 \\
\hline RSS (dBm) & -60.21 & -60.74 & -60.88 & -64.83 & -65.80 & -62.91 \\
\hline $\mathrm{D}(\mathrm{Mb} / \mathrm{S})$ & 2.20 & 2.20 & 2.19 & 2.16 & 2.15 & 2.18 \\
\hline R (km) & 16.50 & 16.00 & 15.80 & 15.04 & 14.62 & 15.34 \\
\hline$P_{B}$ & 0.51 & 0.53 & 0.55 & 0.63 & 0.66 & 0.59 \\
\hline $\boldsymbol{P}_{D}$ & 0.44 & 0.46 & 0.48 & 0.55 & 0.59 & 0.51 \\
\hline BER & $1 \times 10^{-6}$ & $1 \times 10^{-6}$ & $1 \times 10^{-6}$ & $1 \times 10^{-6}$ & $1 \times 10^{-6}$ & $1 \times 10^{-6}$ \\
\hline$\frac{E_{b}}{N_{0}}(d B)$ & 20.39 & 20.87 & 20.91 & 23.05 & 24.16 & 21.28 \\
\hline
\end{tabular}

which are typical of two types of aerial platforms, Low Altitude Platforms (LAP) at $5 \mathrm{~km}$, and High-Altitude Platform (HAP) at $20 \mathrm{~km}$ above the ground.

The coverage radius $\mathrm{R}$ is a function of elevation angles that range between $5^{\circ}$ and $90^{\circ}$ at different altitudes of aerial platform altitudes. The receiver antenna height $h_{r}$ is set at $1.5 \mathrm{~m}$. The total system Bandwidth $B$ is $10 \mathrm{MHz}, \mathrm{R}_{\mathrm{b}}$ is the data rate at $6.048 \mathrm{Mb} / \mathrm{s}$, and the full duplex channel bandwidth is $10.94 \mathrm{kHz} . \eta_{\operatorname{LoS}}$ is an average of $4 \mathrm{~dB}$, whereas $\eta_{N L O S}$ is $10 \mathrm{~dB}$. Table 2 depicts the predicted numerical results produced in 3D Remcom Wireless InSite software tool and MATLAB. Figure 4 through to Fig. 11 visualise these.

Figures 4 and 5 demonstrate the predicted results of the six rescue teams as different colours to highlight the strength of RSS which varies depending on the PL and shadowing effect. RSS decreases with distance and/or shadowing and these get depicted as blue dots, whereas the best RSS get depicted as orange dots. The rescue teams are distributed randomly across a city with a $15 \mathrm{~km}$ footprint radius to cover different situations for both accrued LoS and NLoS conditions, in an effort to obtain realistic predictions for an urban environment. The predicted RSS results of rescue teams float between -57.30 and $-63.81 \mathrm{dBm}$ at an altitude of $5 \mathrm{~km}$, whilst at an altitude of $20 \mathrm{~km}$ they float between -60.21 and $-65.80 \mathrm{dBm}$. At the $5 \mathrm{~km}$ altitude predictions are slightly better than at the $20 \mathrm{~km}$ altitude. The reason for this is the increase in PL at $20 \mathrm{~km}$ due to that high altitude.

Clearly, rescue teams 1, 2, and 3 yield a reasonably good RSS average in comparison to the others rescue teams due to the shadowing effects from obstacles such as highrise buildings. Keeping transmission power constant at different transmitter altitudes yields varying levels of RSS. Based on the predicted RSS obtained in the 3D Remcom Wireless InSite software tool, the rest of the link budget parameters of the six rescue 
Fig. 4 Link budget prediction of an ad hoc network architecture at $5 \mathrm{~km}$

Fig. 5 Link budget prediction of an ad hoc network architecture at $5 \mathrm{~km}$
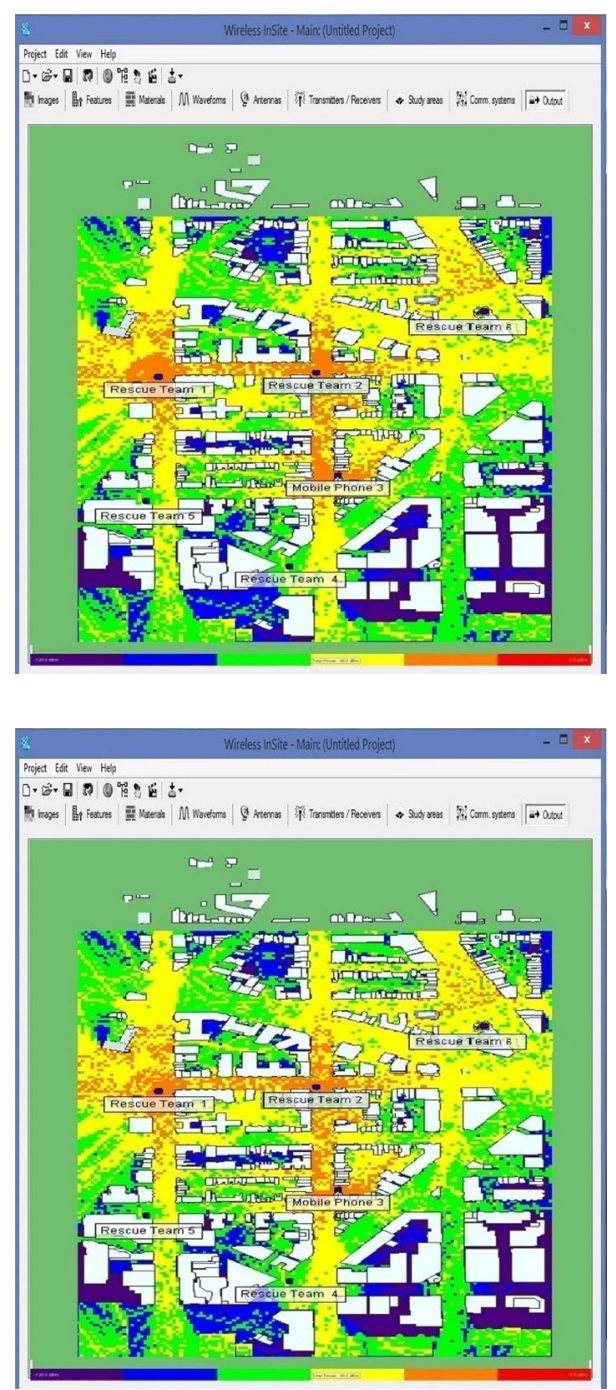

teams have been computed in MATLAB. PL predictions are used for monitoring system performance and coverage to achieve a certain level of reception. PL which is linked to RSS shows a gradual increase as aerial attitudes increase as well as a noticeable affect from shadowing. Thus, the PL at an altitude of $5 \mathrm{~km}$ improves in comparison to $20 \mathrm{~km}$. PL values in both cases are below the maximum allowable PL (MAPL) value of $-134 \mathrm{~dB}$, and range between -128.52 to $-133.5 \mathrm{~dB}$. D decreases with distance as well as with an increasing PL. Thus, D at an altitude of $5 \mathrm{~km}$ yields better predicted results with an average of $14 \%$ improvement in comparison to D at an altitude of $20 \mathrm{~km}$.

Network coverage is affected by transmitter and receiver antenna specifications, geomorphology, and elevation angles. Hence, at an altitude of $5 \mathrm{~km}$ D yields better 


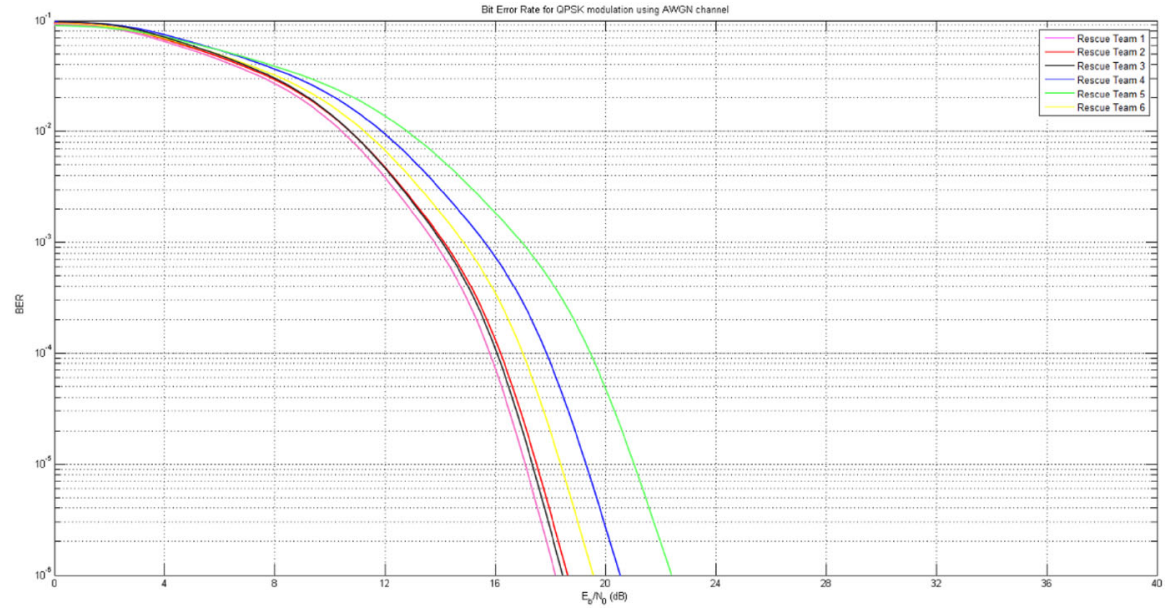

Fig. 6 BER of a signal as a function of $\mathrm{Eb} / \mathrm{No}$ at an altitude of $5 \mathrm{~km}$

predicted $\mathrm{R}$ results with an average of $4 \%$ in comparison to $\mathrm{D}$ at an altitude of $20 \mathrm{~km}$ due to the big difference in altitude. Figures 6 and 7 show the lowest BER and the predicted Eb/No results at altitudes of $5 \mathrm{~km}$ and $20 \mathrm{~km}$, respectively, which are simulated using the "semilogy" function in MATLAB. BER and Eb/No parameters are used interchangeably for monitoring the performance of a digital wireless system and have been considered as two QoS parameters. The best link performance is the one that allows for the lowest possible BER with the lowest possible Eb/No. This describes a robust channel, where a low error rate may be achieved without requiring excessive transmission power. At the lowest BER of $1 \times 10^{-6}$, the aerial platform at an altitude of $5 \mathrm{~km}$ exhibits the lowest $\mathrm{Eb} / \mathrm{No}$ with a range of approximately $2-4 \mathrm{~dB}$ in comparison to the aerial platform at an altitude of $20 \mathrm{~km}$. As the PL decreases, both the BER and $\mathrm{Eb} /$ No decrease, and system performance improves. Varying aerial platform altitudes with an increase in distance across different locations of rescue teams also affects BER and Eb/No. The overall results of these two QoS parameters reveal reasonable predictions. However, an improvement can be achieved in relation to the performance of the QoS parameters which may lead to reduction in the required transmission power from sensors and an improved link performance between aerial platform and ground sensors, thus, increasing the lifetime and performance of the network.

In the event of an emergency situation, network operators experience a much higher than normal volume of data traffic because of the rescue and relief operations as well as all other non-emergency and residential communications. Thus, it is significant to include the GoS to measure the performance of rescue teams via $P_{B}$ and $P_{C}$. Figures 8 and 9 show the probability of blocking as functions of the number of channels and traffic intensity in Erlang for the six rescue teams at an altitude of $5 \mathrm{~km}$ and $20 \mathrm{~km}$ respectively. $P_{B}$ means that a new call arriving is rejected because all servers (channels) are busy. This measures traffic congestion in the telephone network in cases of lost calls. However, Figs. 10 and 11 illustrate the probability of a call being delayed, as a function of the number of channels and traffic intensity in Erlang. $P_{B}$ is calculated 


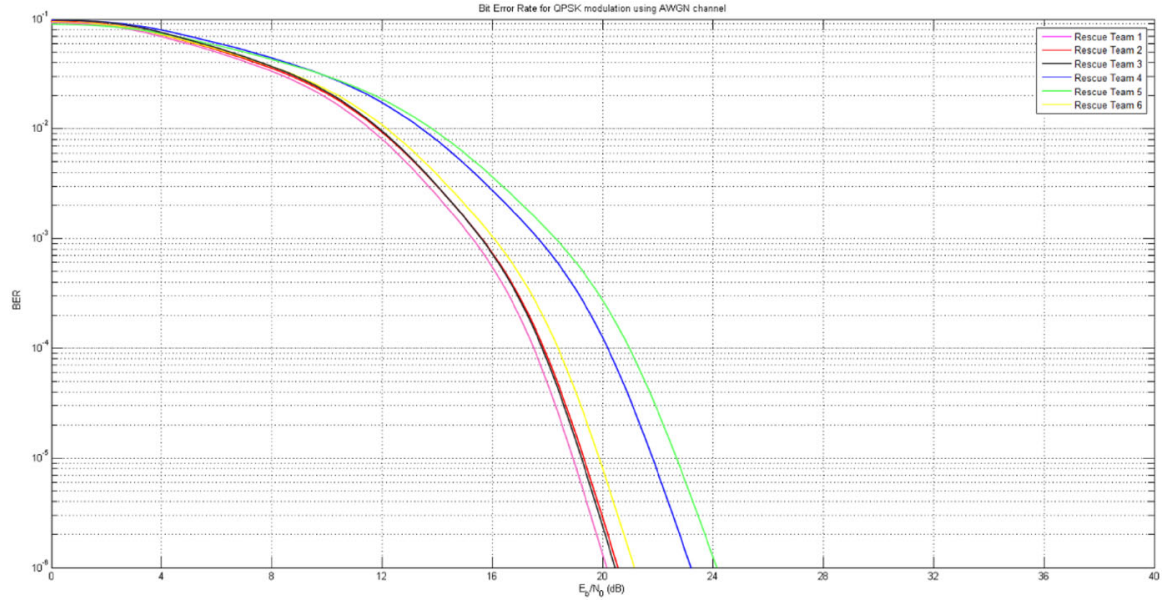

Fig. 7 BER of a signal as a function of Eb/No at an altitude of $20 \mathrm{~km}$

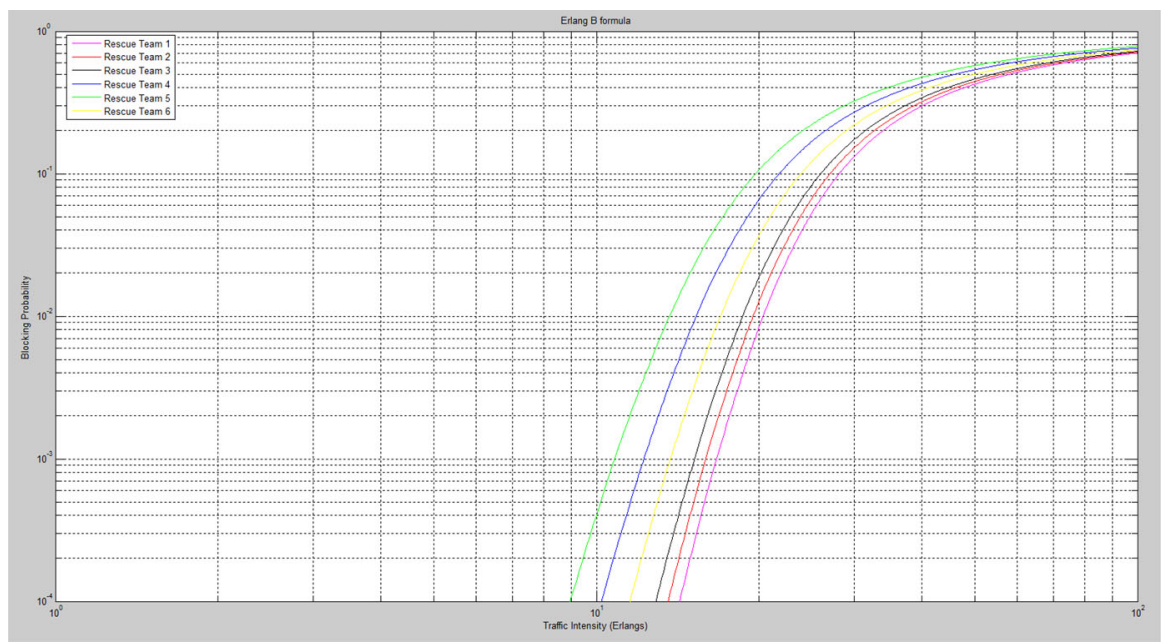

Fig. 8 Erlang B probability of blocking at an altitude of $5 \mathrm{~km}$

as a function of traffic intensity in Erlang of different number of channels multiplied by obtaining the RSS of each rescue team at different aerial platform altitudes. The predicted results show that the average $P_{B}$ in the case of an aerial platform at an altitude of $5 \mathrm{~km}$ are slightly lower than in case of an aerial platform at an altitude of $20 \mathrm{~km}$ due to the smaller number of cells, stronger RSS, less distance and thus less congestion. Thus, the obtained $P_{B}$ values are linked to the RSS, and rescue teams which experience stronger RSS have better $P_{B}$ performance with an average improvement between $2 \%$ and $15 \%$. As the traffic load increases $P_{C}$ increases, which suggests a positive correlation. Thus, the obtained $P_{C}$ values are linked to $P_{B}$, so rescue teams which experience high $P_{B}$ values, also have high $P_{C}$ values. 


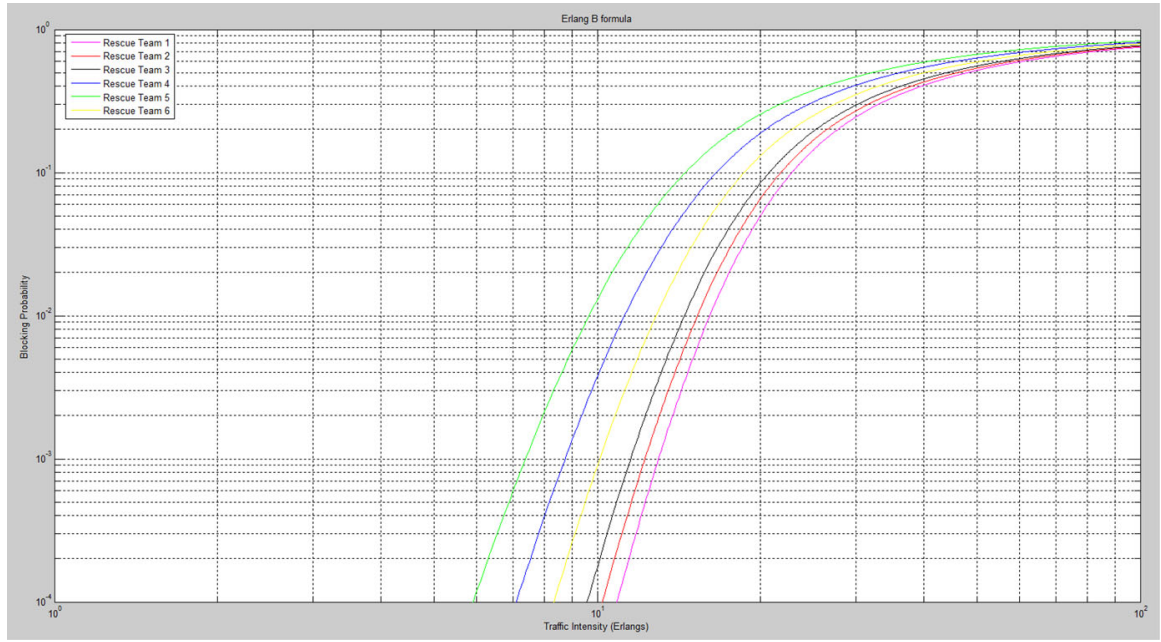

Fig. 9 Erlang B probability of blocking at an altitude of $20 \mathrm{~km}$

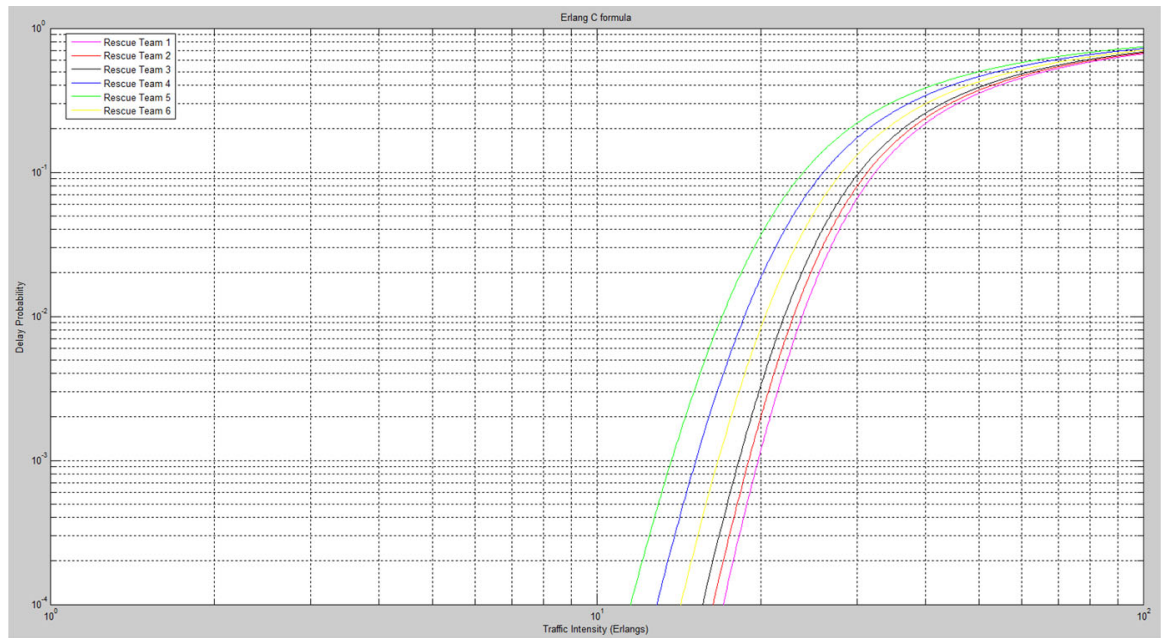

Fig. 10 Erlang C probability of delay at an altitude of $5 \mathrm{~km}$

Figure 12 shows the RBFNN framework in MATLAB. Optimization with RBFNN focuses on giving high priority to specific parameters, which are classified into three groups: the highest priority group includes $R S S, P_{B}$, and $P_{D}$, the second highest priority group includes $B E R$ and $E b / N o$, the lowest priority group includes $P L, D$ and $R$. When RBFNN concludes, the optimized cluster is entered as input to the 3D Remcom Wireless InSite software and MATLAB tools to evaluate the performance of the six rescue teams of the physical model. Figures 13, 14, 15 and 16 illustrate the predictions evolved with the ML framework across selected aerial platform altitudes and elevation angles. Table 3 reports the numerical predictions as they evolve in MATLAB and Figs. 17, 18, 19 and 20 visualise these. 


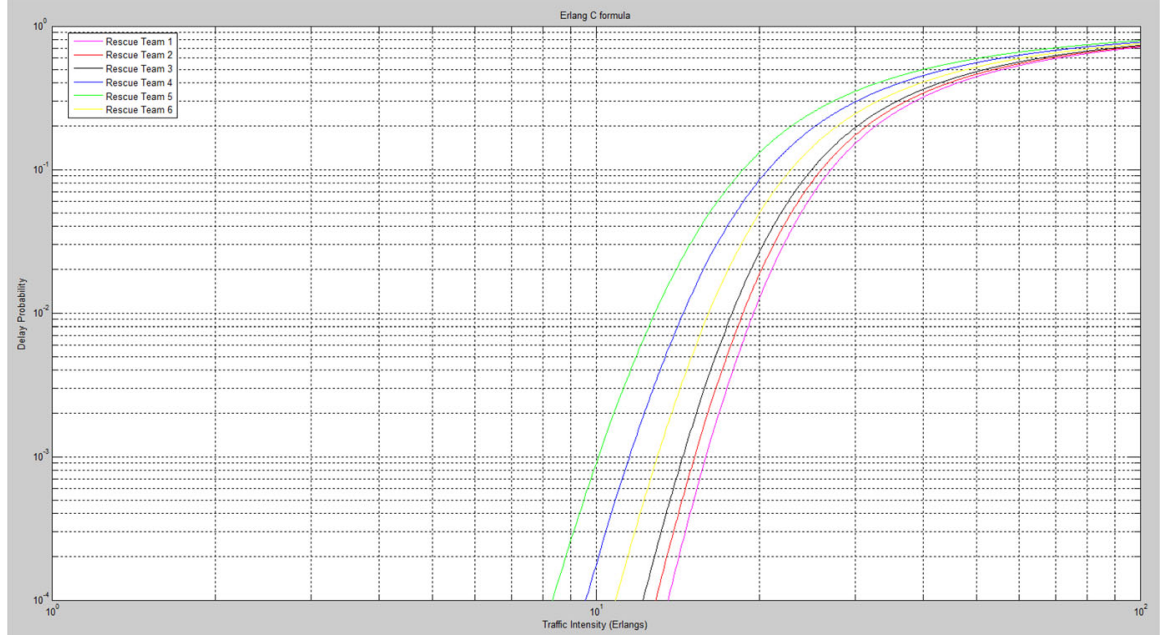

Fig. 11 Erlang C probability of delay at an altitude of $20 \mathrm{~km}$

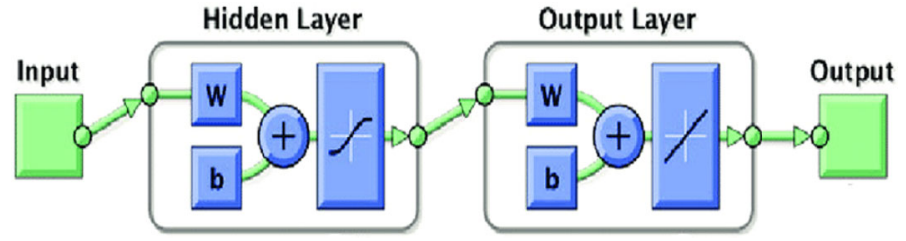

Fig. 12 RBFNN layout in MATLAB

Fig. 13 Regression for RBFNN in MATLAB

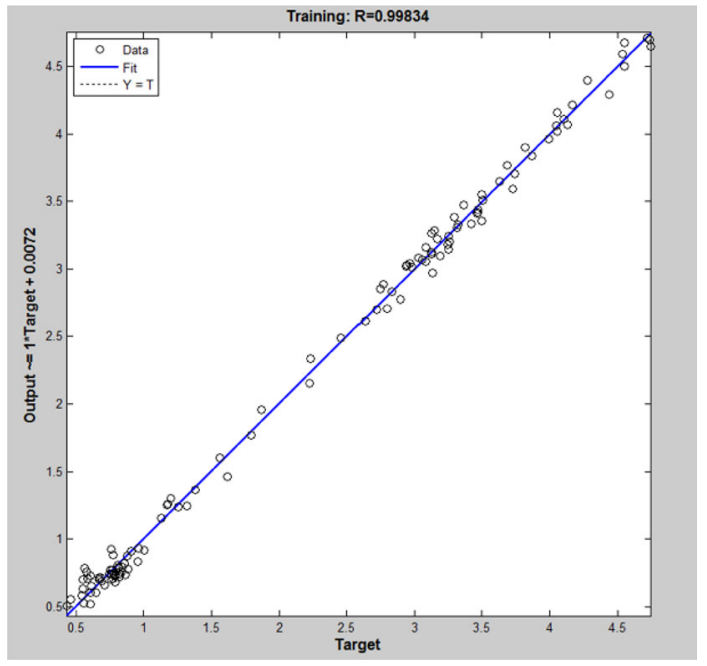




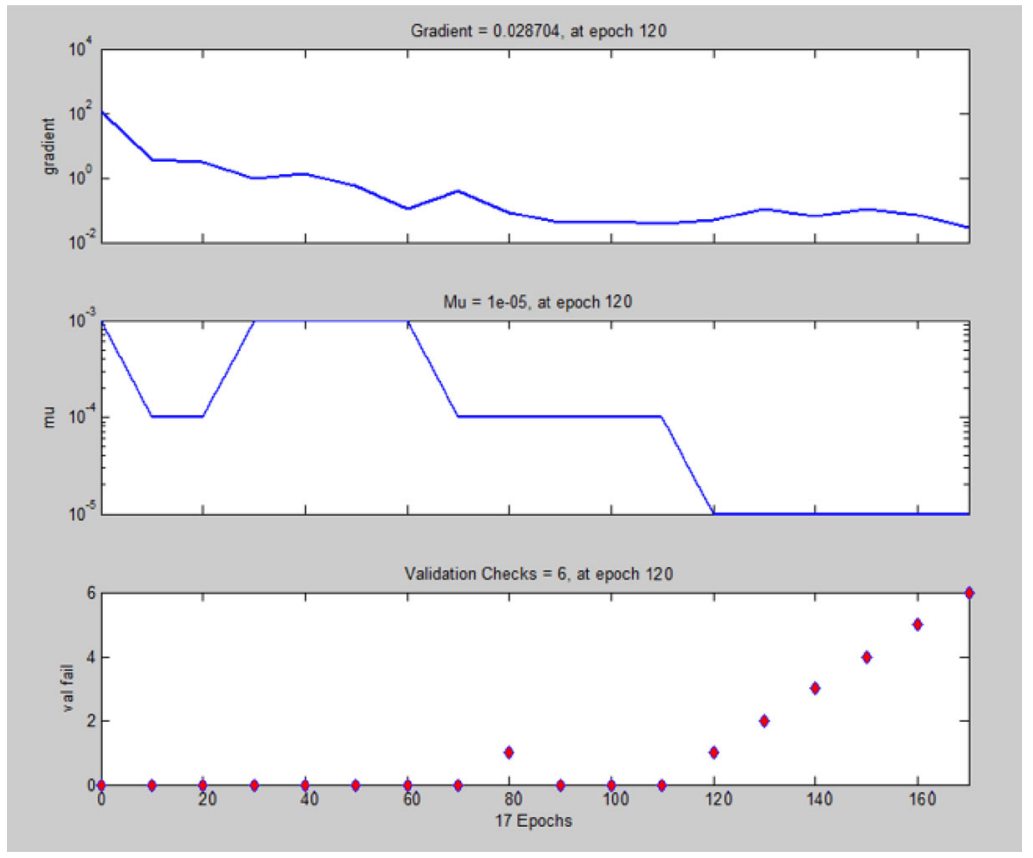

Fig. 14 Training data of RBFNN in MATLAB

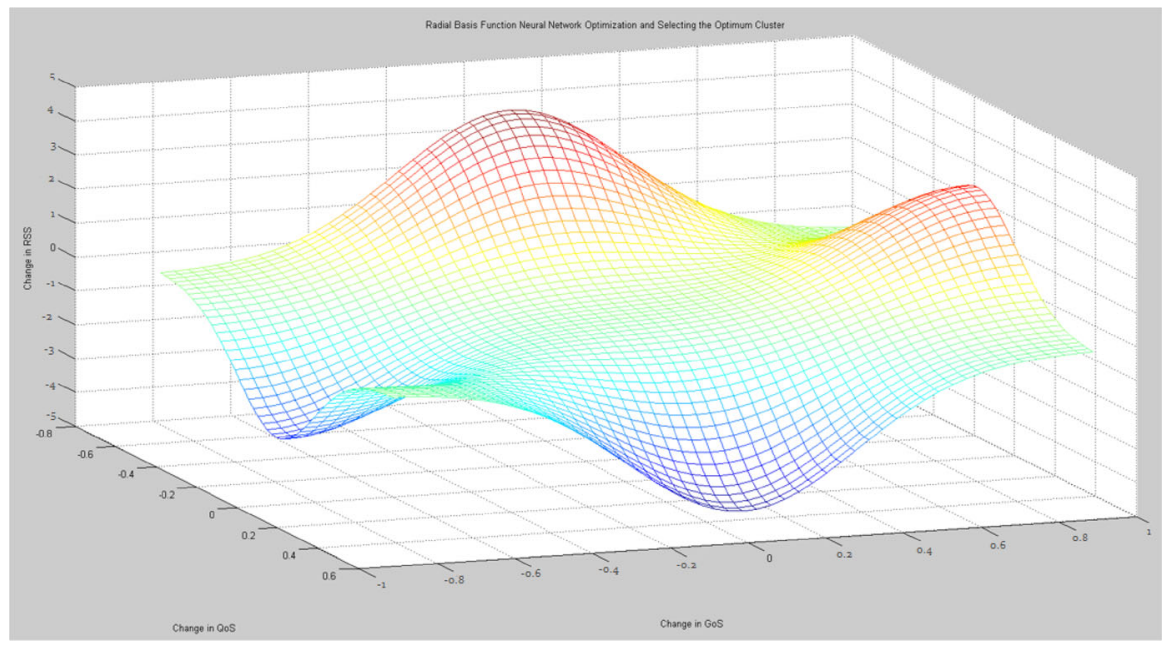

Fig. 15 Gaussian mixture distribution function of the proposed RBFNN in MATLAB

Figure 13 shows a regression plot for RBFNN that gives an indication of how well the variation in the output matches the targets. The solid line represents the perfect fit linear regression line between outputs and targets and the best linear fit is indicated by a dashed line. The $\mathrm{R}$ value is an indication of the relationship between the outputs 
Fig. 16 MSE performance of the proposed RBFNN in MATLAB

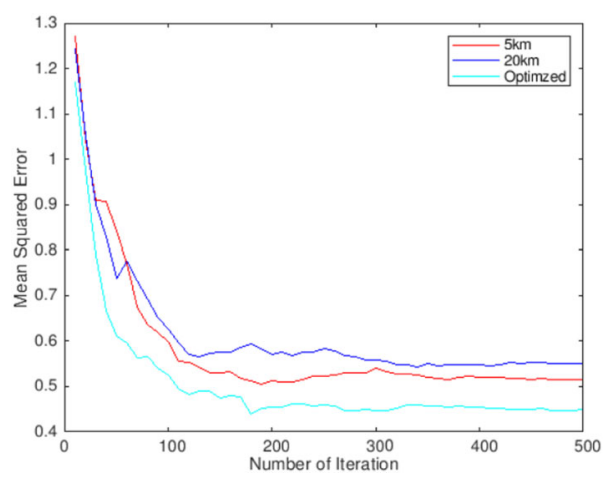

Table 3 Numerical predictions of the proposed RBFNN

\begin{tabular}{|c|c|c|c|c|c|c|}
\hline \multirow[b]{2}{*}{ Parameters } & \multicolumn{6}{|c|}{ Performance of rescue teams for the optimized cluster at $h_{t}=9 \mathrm{~km}$ of $\theta=23^{\circ}$} \\
\hline & Team 1 & Team 2 & Team 3 & Team 4 & Team 5 & Team 6 \\
\hline PL (dB) & -125.28 & -125.41 & -125.9 & -128.69 & -129.78 & -127.28 \\
\hline RSS (dBm) & -56.49 & -57.02 & -57.16 & -61.11 & -62.09 & -59.16 \\
\hline $\mathrm{D}(\mathrm{Mb} / \mathrm{S})$ & 2.37 & 2.37 & 2.35 & 2.33 & 2.31 & 2.34 \\
\hline $\mathrm{R}(\mathbf{k m})$ & 15.00 & 15.00 & 15.00 & 15.00 & 15.00 & 15.00 \\
\hline$P_{B}$ & 0.32 & 0.34 & 0.36 & 0.44 & 0.47 & 0.40 \\
\hline$P_{D}$ & 0.25 & 0.27 & 0.29 & 0.36 & 0.40 & 0.32 \\
\hline BER & $1 \times 10^{-6}$ & $1 \times 10^{-6}$ & $1 \times 10^{-6}$ & $1 \times 10^{-6}$ & $1 \times 10^{-6}$ & $1 \times 10^{-6}$ \\
\hline$\frac{E_{b}}{N_{0}}(d B)$ & 15.93 & 16.41 & 16.44 & 18.59 & 19.70 & 16.82 \\
\hline
\end{tabular}

and targets. The $\mathrm{R}$ value here shows that the training data indicates a good correlation between targets and outputs as $\mathrm{R}$ moves close to 1 . Thus, this is significant to the RBFNN performance.

Figure 14 depicts the 3-layer training state of the RBFNN performance using Stochastic gradient descent (SGD), which is the value of the backpropagation gradient on each iteration in logarithmic scale. At each iteration the weights and biases are updated. The mu is a control parameter that is used to train the network and its' value directly affects the error convergence. The values of gradient coefficient and mu are decreasing as the number of epochs increases until it reaches the lowest local minimum of the goal function at epoch number 120 for values 0.028704 and $1 \mathrm{e}-05$ respectively. Bringing these values to their lowest minimum results in better training and testing of the NN. Both gradient and mu lines become flat or near to zero which indicates termination of the training process. The validation checks denote the number of successive epochs that the validation performance fails to decrease. Furthermore, validation checking is a way to ensure data overfitting. Training stops when the validation parameter reaches the maximum number of validations of 6 at epoch 120 with the minimum gradient decent and $\mathrm{Mu}$ values. A conclusion which can be drawn about the overall performance of the 3-layer training state of RBFNN is that accurate predictions may be obtained, and that no overfitting occurs before its best validation performance occurs. 
Fig. 17 Link budget prediction of the ad hoc network architecture based on the optimized cluster at $9 \mathrm{~km}$
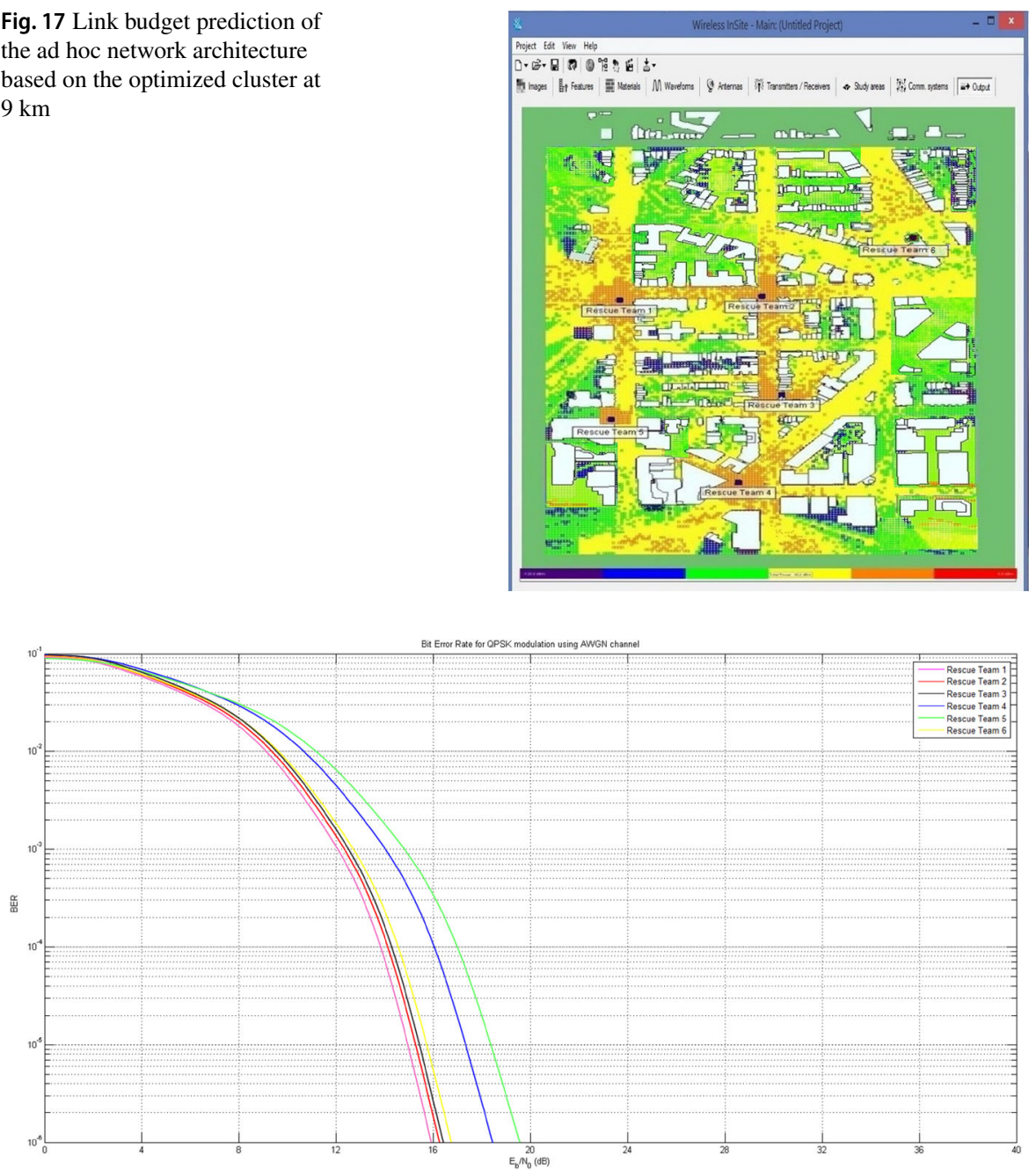

Fig. $18 \mathrm{BER}$ of signal as a function of Eb/No of the optimized cluster at $9 \mathrm{~km}$

Figure 15 shows a Gaussian mixture distribution in multi-dimensions using a shaded mesh plot in MATLAB. Such a distribution can give an understandable description of data that cluster around a mean. The plot of the associated probability density has the Gaussian bell curve peak at the mean and each bell curve corresponds to a different cluster with its own independent mean and covariance. The k-means computes the probability of each cluster's means and covariances, then either updates at every iteration up to the maximum number of iterations, or no significant change in the Gaussian mixture distribution has occurred. Then, the probability density converges to the most suitable clusters that meet our optimizations requirements. The final workspace contains the evolved optimized values. Figure 16 displays the MSE performance of the proposed RBFNN in MATLAB. The performance plot shows the MSE dynamics for 


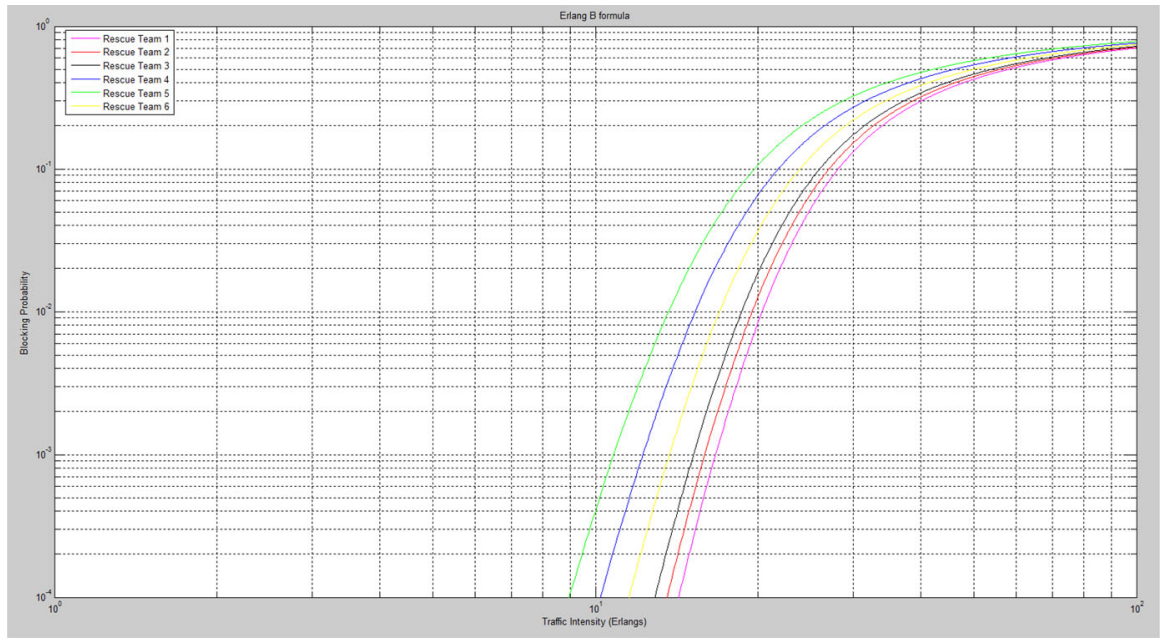

Fig. 19 Erlang B probability of blocking of of the optimized cluster at $9 \mathrm{~km}$

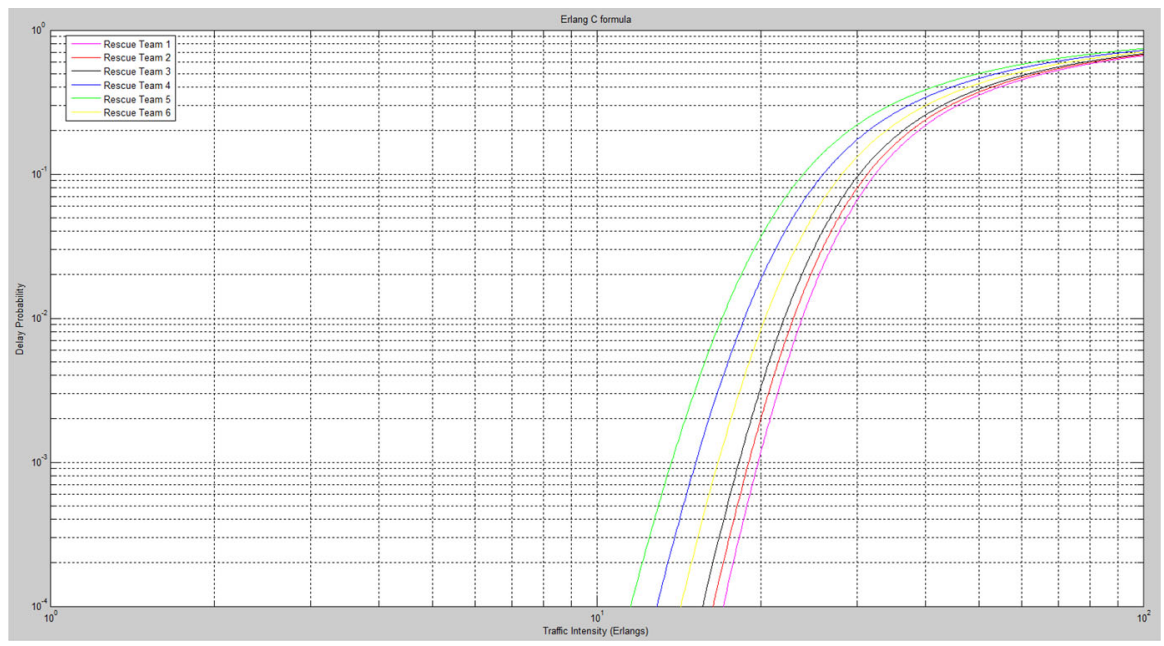

Fig. 20 Erlang C probability of delay of the optimized cluster at $9 \mathrm{~km}$

all datasets in logarithmic scale. Training MSE is decreasing as the number of iterations increase. The plot shows the evolved optimized results were achieved when the RBFNN converges towards the best-fit value at around 120 iterations with a much lower MSE than those of the non-optimized models. The result is fitting and reasonable because, at first, the final MSE is small and, second, the NN converges and cuts off training once the error rate becomes constant after a set number of iterations.

Table 3 shows that the evolved optimized RSS for the six rescue teams yield better predictions in comparison to the non-optimized by an average of $3 \mathrm{dBm}$ to $4.2 \mathrm{dBm}$. Likewise, the evolved optimized PL exhibit similar improvements by an average of 3-4.2 dB as they exhibit a positive correlation. Achieving a higher RSS results in a 
lower PL, which in turns leads to improved coverage and reduced power consumption. Rescue teams 1, 2, and 3 yield a reasonably good RSS average in comparison to the other rescue teams due to shadowing effects from obstacles such as high-rise buildings. However, a noticeable improvement in many areas in the urban environment was an RSS rate well above the acceptable rate of $-80 \mathrm{dBm}$ for the non-optimized. This was the result of meeting environmental requirements and reducing the shadowing effect. The evolved optimized D exhibits an average improvement ranging between 5 and $8 \%$ in comparison to the non-optimized predictions, which increases data rates. The evolved optimized $\mathrm{R}$ is kept constant at $15 \mathrm{~km}$ in order to maintain LoS connectivity and wider footprint coverage. In relation to $P_{B}$ and $P_{D}$, the table reports improvement which is due to two main factors, i.e. the higher RSS and D values, which increase system capacity and grade of service thus with a smaller number of channels being blocked or delayed. The Eb/No and BER values suggest a positive correlation in comparison to non-optimized values, which in turns leads to reduced energy consumption that results in improved QoS.

Using the evolved optimized predictions that we have obtained from the proposed RBFNN, we run the 3D Remcom Wireless InSite simulation to measure the RSS performance of the six rescue teams as Fig. 17 shows. We then re-test the performance of the remainder evolved parameters in MATLAB. Figure 18 displays the predicted lowest BER and Eb/No results for the optimal model, which has been simulated using the "semilogy" function in MATLAB.

At the lowest BER of $1 \times 10^{-6}$, the plot exhibits the lowest Eb/No with its range floating between 20 and $22 \%$ which contributes to optimal performance. It is observed that as PL decreases, both BER and Eb/No decrease, and system performance improves. The overall optimized predictions of these two QoS parameters show reasonable values, which may lead to reduction in the required transmission power from sensors and an improved link performance between aerial platforms and ground sensors, thus, increasing the lifetime and performance of the network. The best link performance is that which allows for the lowest possible BER with the lowest possible $\mathrm{Eb} /$ No. This makes up a robust channel, where one can achieve a low error rate without requiring an increased transmission power.

Figures 19 and 20 illustrate the probabilities of blocking and of a call being delayed as functions of the number of channels and traffic intensity for the six rescue teams in the optimised model. The $P_{B}$ and $P_{C}$ values in the optimised model are lower than those in the non-optimised models with average range between 38 and $40 \%$ because of improved RSS and PL predictions. The evolved $P_{B}$ and $P_{C}$ values are linked to RSS, so rescue teams which experience stronger RSS produce better performance.

\section{Proof of concept development: ad hoc case}

The hurricane that struck Florida in September 2017 [1, 3] highlights how vulnerable terrestrial networks are to both man-made and natural disasters. The series of transmission towers that make up the terrestrial systems infrastructure is largely ground-based 
which puts them directly in the line of danger when it comes to any type of disaster. Furthermore, the limited coverage range of terrestrial towers may not justify the effort required to restore one or more terrestrial towers in the immediate aftermath of a disaster. Deploying an ad hoc network of aerial platforms over the skies of the affected area will swiftly bridge the coverage gaps through a soft infrastructure, a fast start-up time, and on-demand capacity assignment [63, 64]. Furthermore, the low cost and speed of deployment of aerial platforms in comparison to that of satellites will quickly restore connectivity for the hundreds of thousands of victims and users in the disaster zones that lost their connection and without high energy consumption. Furthermore, the use of aerial platforms may help with restoring communications selectively, for example, replacing both overloaded and broken terrestrial links, or around hospitals and emergency rooms or in areas where rescue teams are operating [63-66].

The rest of this section expands on a specific emergency plan by the Vodafone Foundation Instant Network that is sourced from the emergency operations in the state of Florida when it was struck by Hurricane Irma in September 2017 and wired and wireless communication links between residents and rescue teams were wrecked [67]. Under the light of that plan, six rescue teams are distributed randomly in an urban environment for disaster relief operations and are served by an aerial platform system occupying the altitudes of $5 \mathrm{~km}$ and $20 \mathrm{~km}$. At each altitude eight parameters (PL, $\mathrm{RSS}, \mathrm{D}, \mathrm{R}, \mathrm{BER}$ Eb/No, $\mathrm{P}_{\mathrm{B}}$, and $\mathrm{P}_{\mathrm{C}}$ ) are simulated in MATLAB. Then, the parameter predictions obtained are used as the input vector into the RBFNN before comparing them against the simulated predictions of Sect. 5. Table 4 shows the performance of the six rescue teams under both non-optimized and optimised performance produced by RBFNN based on the Vodafone Instant Network plan for the Irma emergency operations. Figures 21 plots the average numerical predictions of the optimised model produced by RBFNN against the non-optimized based on the Vodafone Network for Irma at different aerial platform altitudes and elevation angles.

The simulation results of Table 4 and Fig. 21 show that there are many factors that may affect the performance of each rescue team's parameters: geomorphology, shadowing, altitudes, elevation angle, coverage footprint and LoS connectivity. The optimized RSS for the six rescue teams produce better predictions in comparison to the non-optimized by an average of $7 \mathrm{dBm}$, which in turns leads to improved coverage and reduced power consumption. The evolved optimized D exhibits an average improvement of $6 \%$ in comparison to the non-optimized predictions, which yields increased data rates. The evolved optimized $\mathrm{R}$ exhibits an enhancement ranging between 8 and $11 \mathrm{~km}$. In terms of the optimized $P_{B}$ and $P_{D}$ the table reports improvements due to higher RSS and D, which in turn increases system capacity and GoS with only a smaller number of channels are being blocked or delayed. The obtained Eb/No and BER values suggest a positive correlation in comparison to non-optimized values, which in turns leads to a reduction in energy consumption and improved QoS.

The overall performance of the RFBNN framework has shown much promise during a simulated rapid deployment of an aerial platform system in the immediate aftermath of an emergency. The ATG propagation model has been simulated in relation to path 
Table 4 Numerical predictions based on the Vodafone Network plan during Irma

\begin{tabular}{|c|c|c|c|c|c|c|}
\hline \multirow[b]{2}{*}{ Parameters } & \multicolumn{6}{|c|}{ Performance of rescue teams for $h_{t}=5 \mathrm{~km}$ of $\theta=15^{\circ}$} \\
\hline & Team 1 & Team 2 & Team 3 & Team 4 & Team 5 & Team 6 \\
\hline PL (dB) & -133.18 & -125.70 & -130.43 & -129.13 & -132.14 & -134 \\
\hline RSS (dBm) & -66.89 & -57.66 & -62.13 & -61.84 & -64.71 & -67.50 \\
\hline $\mathrm{D}(\mathrm{Mb} / \mathrm{S})$ & 3.11 & 3.44 & 3.22 & 3.28 & 3.16 & 3.09 \\
\hline $\mathrm{R}(\mathrm{km})$ & 34.20 & 27.56 & 31 & 29.76 & 33.42 & 35.09 \\
\hline $\boldsymbol{P}_{B}$ & 0.56 & 0.49 & 0.53 & 0.52 & 0.55 & 0.57 \\
\hline $\boldsymbol{P}_{D}$ & 0.45 & 0.40 & 0.43 & 0.42 & 0.44 & 0.46 \\
\hline BER & $1 \times 10^{-6}$ & $1 \times 10^{-6}$ & $1 \times 10^{-6}$ & $1 \times 10^{-6}$ & $1 \times 10^{-6}$ & $1 \times 10^{-6}$ \\
\hline$\frac{E_{b}}{N_{0}}(d B)$ & 22.78 & 15.07 & 19.45 & 18.88 & 21.64 & 24.50 \\
\hline \multicolumn{7}{|c|}{ Performance of rescue teams for $h_{t}=20 \mathrm{~km}$ of $\theta=30^{\circ}$} \\
\hline PL (dB) & -138.15 & -130.76 & -134.68 & -133.41 & -136.95 & -139.40 \\
\hline RSS (dBm) & -71.21 & -64.07 & -67.33 & -66.18 & -69.48 & -72.13 \\
\hline $\mathrm{D}(\mathrm{Mb} / \mathrm{S})$ & 3.22 & 3.32 & 3.27 & 3.28 & 3.25 & 3.21 \\
\hline R (km) & 55.87 & 50.68 & 53.46 & 52.05 & 54 & 57.43 \\
\hline$P_{B}$ & 0.65 & 0.59 & 0.61 & 0.61 & 0.63 & 0.66 \\
\hline$P_{D}$ & 0.58 & 0.51 & 0.55 & 0.54 & 0.56 & 0.59 \\
\hline BER & $1 \times 10^{-6}$ & $1 \times 10^{-6}$ & $1 \times 10^{-6}$ & $1 \times 10^{-6}$ & $1 \times 10^{-6}$ & $1 \times 10^{-6}$ \\
\hline$\frac{E_{b}}{N_{0}}(d B)$ & 26.19 & 17.34 & 22.55 & 20.67 & 24.84 & 27.02 \\
\hline \multicolumn{7}{|c|}{ Performance of rescue teams for the optimized cluster at $h_{t}=12 \mathrm{~km}$ of $\theta=16^{\circ}$} \\
\hline PL (dB) & -129.07 & -121.89 & -124.34 & -124 & -127.50 & -130.60 \\
\hline RSS (dBm) & -61.11 & -53.92 & -57.55 & -56.12 & -59.43 & -62.09 \\
\hline $\mathrm{D}(\mathrm{Mb} / \mathrm{S})$ & 3.58 & 3.84 & 3.75 & 3.77 & 3.65 & 3.55 \\
\hline $\mathbf{R}(\mathbf{k m})$ & 62.87 & 51.33 & 55.63 & 54.79 & 59.88 & 64.42 \\
\hline$P_{B}$ & 0.48 & 0.38 & 0.41 & 0.40 & 0.46 & 0.49 \\
\hline$P_{D}$ & 0.41 & 0.33 & 0.37 & 0.36 & 0.39 & 0.42 \\
\hline BER & $1 \times 10^{-6}$ & $1 \times 10^{-6}$ & $1 \times 10^{-6}$ & $1 \times 10^{-6}$ & $1 \times 10^{-6}$ & $1 \times 10^{-6}$ \\
\hline$\frac{E_{b}}{N_{0}}(d B)$ & 22.76 & 16.03 & 19.63 & 18.99 & 21.28 & 23.05 \\
\hline
\end{tabular}

loss, RSS, frequency, transmitter and receiver height, antenna gains, throughput, and coverage. This is shown to help with increasing wireless connectivity and filling any coverage gaps between rescue teams, emergency departments, headquarters, and of course disaster victims as well as optimising link budget performance. Furthermore, the framework optimizes the GoS and QoS performance of the aerial platform system, thereby increasing system capacity, improving connectivity and reducing energy consumption. Figure 22 plots training data against validation data both sourced from the Vodafone Network.

\section{Concluding discussion}

Establishing of an ad hoc but robust wireless communication service for disaster relief management in place of a disrupted terrestrial system with the aid of machine learning has been the major motivation of this paper. Reliable telecommunication systems in the immediate aftermath of a catastrophic event makes such a huge difference in the combined effort by local authorities, local fire and police departments, the Red 


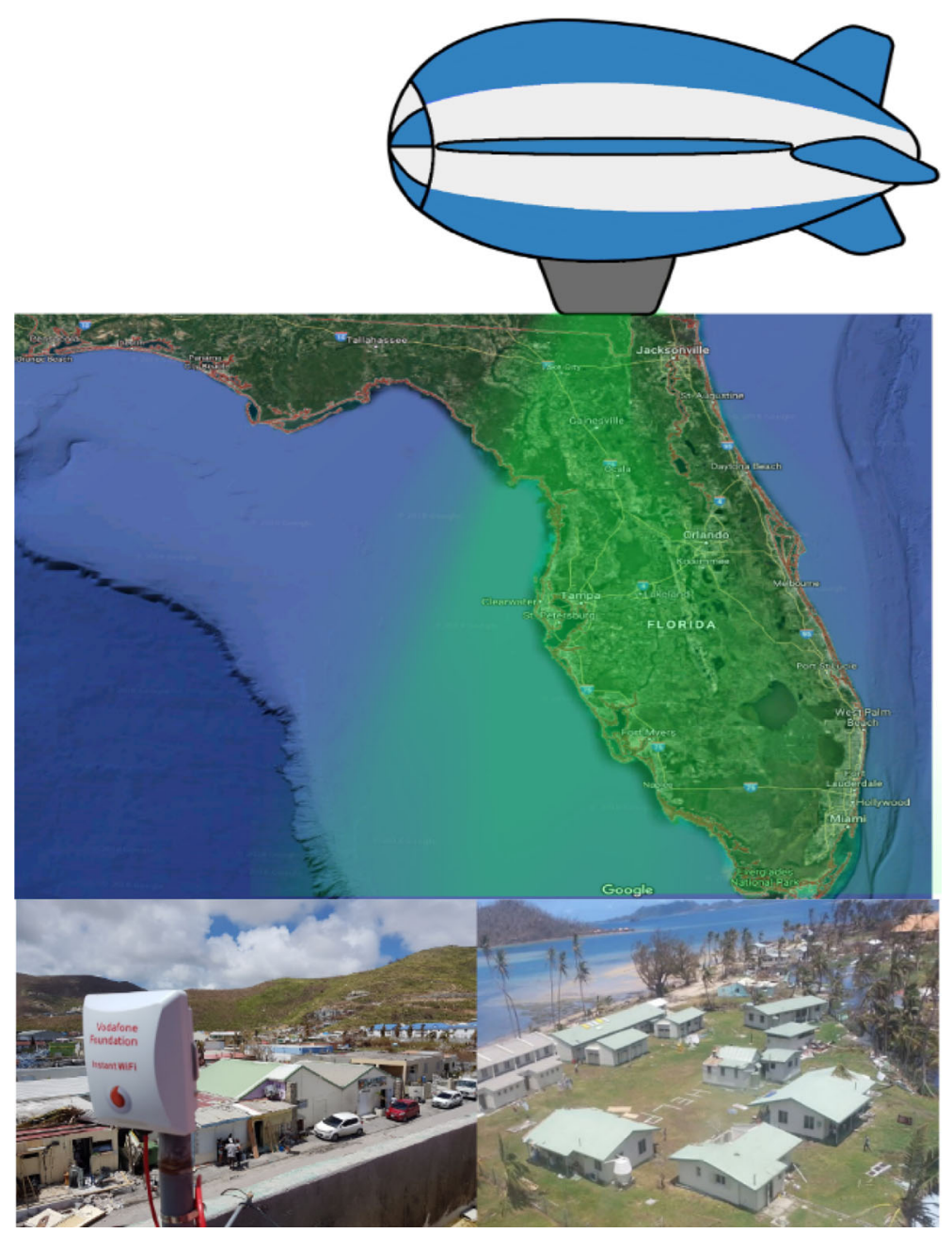

Fig. 21 Aerial platform over Florida state with screenshot of Vodafone Network

Cross, and search and rescue teams in their efforts to save lives. Crisis and emergency situations usually result in chaos and having a reliable communications network to support such efforts and to broadcast vital information and advice to victims seeking refuse and rescue is life-critical. Our proposed evolved ATG propagation model to be used with the aerial platform system has been simulated to provide connectivity for our six rescue teams and its overall performance shows robustness in restoring communication links during such emergency relief operations. As a propagation model, it offers improved optimised link budget performance in terms of PL, RSS, D, and R, which yields higher LoS connectivity, wider coverage and increased number of users. Including $\mathrm{P}_{\mathrm{B}}, \mathrm{P}_{D}$, and $\mathrm{Eb} / \mathrm{No}$ in the evolved model yields increased system capacity 


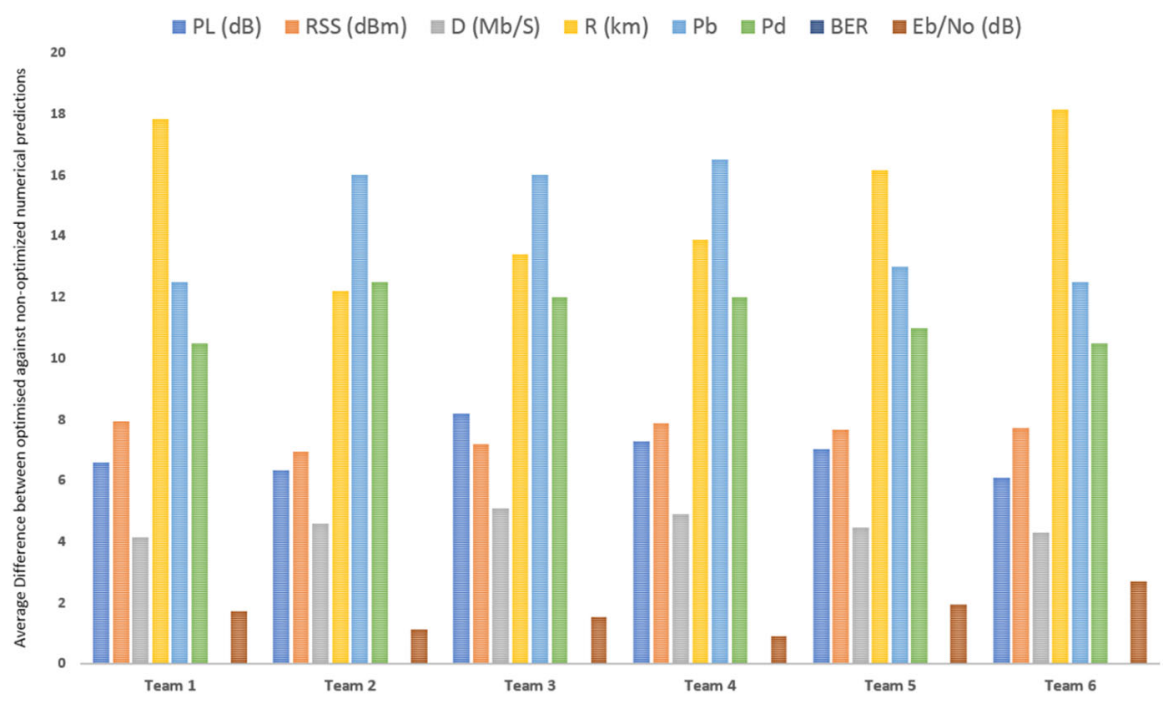

Fig. 22 Comparison between optimised and non-optimized predictions based on the Vodafone Network for Irma

alongside a reduced energy consumption. As future work, considering geolocations may help include and fine-tune environmental considerations.

Open Access This article is distributed under the terms of the Creative Commons Attribution 4.0 International License (http://creativecommons.org/licenses/by/4.0/), which permits unrestricted use, distribution, and reproduction in any medium, provided you give appropriate credit to the original author(s) and the source, provide a link to the Creative Commons license, and indicate if changes were made.

\section{References}

1. Staletovich J (2017) Irma damage expected to reach $\$ 18$ billion in the U.S. Miamiherald. http://www. miamiherald.com/news/weather/hurricane/article173182856.html. Accessed Nov 2018

2. Wzorek M, Berger C, Rudol P, Doherty P (2018) Deployment of ad hoc network nodes using UAVs for search and rescue missions. In: International electrical engineering congress, pp 1-4

3. Hurricane Irma Florida State (2017) Federal Communications Commission. https://www.fcc.gov/irma. Accessed Nov 2018

4. Wang Y, Xu Y, Zhang Y, Zhang P (2017) Hybrid satellite-aerial-terrestrial networks in emergency scenarios: a survey. IEEE China Commun 14(7):1-13

5. Wng Y, Xu Y, Zhang Y, Zhang P (2016) Aerial base stations with opportunistic links for next generation emergency communications. IEEE Commun Mag 54(4):31-39

6. Chandrasekharan S, Gomez K, Al-Hourani A, Kandeepan S, Rasheed T, Goratti L, Reynaud L, Grace D, Bucaille I, Wirth T, Allsopp S (2016) Designing and implementing future aerial communication networks. IEEE Commun Mag 54(5):26-34

7. Yuniarti D (2018) Regulatory challenges of broadband communication services from high altitude platforms (HAPs). In: International conference on information and communications technology (ICOIACT), pp 919-922

8. Li B, Fei Z, Zhang Y (2019) UAV communications for $5 \mathrm{G}$ and beyond: recent advances and future trends. IEEE Internet Things J 6(2):2241-2263

9. Mozaffari M, Saad W, Bennis M, Nam Y, Debbah M (2019) A tutorial on UAVs for wireless networks: applications, challenges, and open problems. In: IEEE communications surveys \& tutorials, p 1 
10. Almalki FA, Angelides MC (2019) Evolution of an optimal propagation model for the last mile with low altitude platforms using machine learning. Elsevier Comput Commun J 142-143:9-33

11. Almalki FA, Angelides MC (2016) Considering near space platforms to close the coverage gap in wireless communications; the case of the Kingdom of Saudi Arabia. In: FTC 2016-future technologies conference, pp 224-230

12. Hadiwardoyo S, Calafate C, Cano J, Ji Y, Hernandez-Orallo E, Manzoni P (2019) 3D simulation modeling of UAV-to-car communications. IEEE Access 7:8808-8823

13. Helmy M, Baykas T, Arslan H (2015) Optimization of aerial base station location in LAP for disaster situations. In: IEEE conference on standards for communications and networking (CSCN), Institute of Electrical and Electronics Engineers (IEEE), pp 240-244

14. Aziz I, Iskandar (2014) Disaster mitigation techniques based on LTE release 8 network employed using HAPS. In: 8th International conference on telecommunication systems services and applications (TSSA), pp 1-6

15. Kandeepan S, Gomez K, Rasheed T, Reynaud L (2011) Energy efficient cooperative strategies in hybrid aerial-terrestrial networks for emergencies. In: IEEE 22nd international symposium on personal indoor and mobile radio communications (PIMRC), Toronto, Canada, pp 294-299

16. Ranjan A, Panigrahi B, Rath H, Misra P, Simha A, Sahu H (2018) A study on pathloss model for UAV based urban disaster and emergency communication systems. In: Twenty fourth national conference on communications (NCC), Hyderabad, India, pp 1-6

17. Zhao $\mathrm{N}$ et al (2019) UAV-assisted emergency networks in disasters. IEEE Wirel Commun 26(1):45-51

18. Chandrasekharan S, Al-Hourani A, Gomez K, Kandeepan S, Evans R, Reynaud L, Scalise S (2016) Performance evaluation of LTE and WiFi technologies in aerial networks. IEEE Globecom workshops (GC Wkshps), Washington, USA, pp 1-7

19. Hariyanto H, Santoso H, Widiawan A (2009) Emergency broadband access network using low altitude platform. In: International conference on instrumentation, communication, information technology, and biomedical engineering, pp 1-8

20. Zhang Q, Jiang M, Feng Z, Li W, Zhang W, Pan M (2019) IoT enabled UAV: network architecture and routing algorithm. IEEE Internet Things J 6:3727-3742

21. Gomez K, Rasheed T, Reynaud L, Kandeepan S (2013) On the performance of aerial LTE base-stations for public safety and emergency recovery. IEEE Globecom workshops, pp 1391-1396

22. Sae J, Yunas S, Lempiainen J (2016) Coverage aspects of temporary LAP network. In: 12th annual IEEE conference on wireless on-demand network systems and services (WONS), pp 1-4

23. Alsamhi S, Ma O, Ansari M, Gupta S (2019) Collaboration of drone and internet of public safety things in smart cities: an overview of QoS and network performance optimization. Drones 3(1):13

24. Motlagh N, Taleb T, Arouk O (2016) Low-altitude unmanned aerial vehicles-based internet of things services: comprehensive survey and future perspectives. IEEE Internet Things J 3(6):899-922

25. Yang Z, Mohammed A (2011) Deployment and capacity of mobile WiMAX from high altitude platform. In: 2011 IEEE vehicular technology conference (VTC Fall), pp 1-5

26. Qiantori A, Sutiono A, Hariyanto H, Suwa H, Ohta T (2012) An emergency medical communications system by low altitude platform at the early stages of a natural disaster in Indonesia. J Med Syst 36(1):41-52

27. Gunaratna G, Jayarathna P, Sandamini S, De Silva D (2015) Implementing wireless adhoc networks for disaster relief communication. In: 8th international conference on ubi-media computing (UMEDIA), pp 1-6

28. Reynaud L, Rasheed T, Kandeepan S (2011) An integrated aerial telecommunications network that supports emergency traffic. In: 14th International symposium on wireless personal multimedia communications (WPMC), pp 1-5

29. Dalmasso I, Galletti I, Giuliano R, Mazzenga F (2012) WiMAX networks for emergency management based on UAVs. In: IEEE first AESS European conference on satellite telecommunications, pp 1-6

30. Gomez K, Hourani A, Goratti L, Riggio R, Kandeepan S, Bucaille I (2015) Capacity evaluation of aerial LTE base-stations for public safety communications. In: IEEE European conference on networks and communications (EuCNC), pp 133-138

31. Katzis K, Grondoudis A (2011) Disaster monitoring and disaster relief using high altitude platforms. In: 1st International conference in safety crisis management, At Nicosia, pp 1-4

32. Alsamhi S, Ma O, Ansari M, Almalki F (2019) Survey on collaborative smart drones and internet of things for improving smartness of smart cities. IEEE Access 7:128125-128152 
33. Vinogradov E, Sallouha H, Bast S, Azari M, Pollin S (2018) Tutorial on UAVs: a blue sky view on wireless communication. J Mob Multimed 14(4):395-468

34. Abrajano G (2017) Demonstrations of post-disaster resilient communications and decision-support platform with UAVs, ground teams and vehicles using delay-tolerant information networks on sub$\mathrm{GHz}$ frequencies. In: IEEE global humanitarian technology conference (GHTC), pp 1-8

35. Wang Y, Xu Y, Zhang Y, Zhang P (2017) Hybrid satellite-aerial-terrestrial networks in emergency scenarios: a survey. China Commun 14(7):1-13

36. Allsopp S (2014) Emergency airborne 4G comms to aid disaster traffic management. In: Road transport information and control conference (RTIC 2014), pp 1-7

37. Vodafone Instant Network. http://www.vodafone.com/content/foundation/instant-network-emergency. html. Accessed Nov 2018

38. Huang J, Wang Y, Lien Y (2013) Deployment scheduling of contingency cellular network for disaster relief operations. 15th Asia-Pacific network operations and management symposium (APNOMS), pp $1-6$

39. Kosmerl J, Vilhar A (2014) Base stations placement optimization in wireless networks for emergency communications. In: IEEE international conference on communications workshops (ICC), pp 200-205

40. Bucaille I, Hethuin S, Rasheed T, Munari A, Hermenier R, Allsopp S (2013) Rapidly deployable network for tactical applications: aerial base station with opportunistic links for unattended and temporary events ABSOLUTE example. In: IEEE military communications conference, pp 1116-1120

41. Habib A, ul-Islam Q (2013) MIMO channel modeling for integrated high-altitude platforms, geostationary satellite/land mobile satellite and wireless terrestrial networks. J Space Technol 3(1):19-26

42. Vilhar A, Hrovat A, Javornik T, Mohorcic M (2013) Experimental analysis of wireless temporary networks deployed by low altitude platforms. IEEE 18th international workshop on computer aided modeling and design of communication links and networks (CAMAD), pp 238-242

43. Zhang S, Wang Z, Qiu M, Liu M (2013) BER-based power scheduling in wireless sensor networks. J Signal Process Syst 72(3):197-208

44. Poulakis M, Vassaki S, Panagopoulos A (2013) Satellite-based wireless sensor networks: radio communication link design. In: 7th European conference on antennas and propagation, pp 2620-2624

45. Albagory Y, Said O (2015) Performance enhancement of high-altitude platforms wireless sensor networks using concentric circular arrays. AEU Int J Electron Commun 69(1):382-388

46. Singh R, Thompson M, Mathews S, Agbogidi O, Bhadane K, Namuduri K (2017) Aerial base stations for enabling cellular communications during emergency situation. In: International conference on vision, image and signal processing (ICVISP), pp 103-108

47. Saha S, Nandi S, Paul P, Shah V, Roy A, Das S (2015) Designing delay constrained hybrid ad hoc network infrastructure for post-disaster communication. Ad Hoc Netw 25(B):406-429

48. Zhang S, Liu J (2018) Analysis and optimization of multiple unmanned aerial vehicle-assisted communications in post-disaster areas. IEEE Trans Veh Technol 67(12):12049-12060

49. Nalineswari D, Rakesh N (2015) Link budget analysis on various terrains using IEEE 802.16 WIMAX standard for $3.5 \mathrm{GHz}$ frequency. In: IEEE international conference on electrical, computer and communication technologies (ICECCT), pp 1-5

50. Hasirci Z, HakkiCavdar I (2012) Propagation modeling dependent on frequency and distance for mobile communications via high altitude platforms (HAPs). In: 35th International conference on telecommunications and signal processing, pp 287-291

51. Al-Hourani A, Kandeepan S, Jamalipour A (2014) Modeling air-to-ground path loss for low altitude platforms in urban environments. In: IEEE global communications conference, pp 2898-2904

52. Almalki FA, Angelides MC (2017) Empirical evolution of a propagation model for low altitude platforms. Comput Conf 2017:1297-1304

53. Said O, Albagory Y (2016) Internet of things-based free learning system: performance evaluation and communication perspective. IETE J Res 63(1):31-44

54. Almalki F, Angelides M (2017) Propagation modelling and performance assessment of aerial platforms deployed during emergencies. In: 12th IEEE international conference for internet technology and secured transactions (ICITST-2017), pp 238-243

55. Agbinya J, Aguayo-Torres M, Klempous R (2013) 4G wireless communication networks: design planning and applications. River Publishers, Aalborg

56. Deaton J (2008) High altitude platforms for disaster recovery: capabilities, strategies, and techniques for emergency telecommunications. EURASIP J Wirel Commun Netw 2008:31-44 
57. Airspan networks, leaders in $4 \mathrm{G}$ technology. http://www.airspan.com/about-airspan/. Accessed $10 \mathrm{Jan}$ 2019

58. Omae M, Ndungu E, Kibet $\mathrm{P}$ (2012) Artificial intelligence approach to signal propagation modeling for outdoor to indoor wireless communication networks; a proposed study. In: Mechanical engineering conference on sustainable research and innovation, Juja, Kenya, pp 289-299

59. Zhao M, Wang Y, Du S, Yin T (2016) The study of rapid localization algorithm from beam scanning by single satellite based on neural network. In: First IEEE international conference on computer communication and the internet (ICCCI), Wuhan, China, pp 186-190

60. Zhang M, Wang X, Liu M (2005) Adaptive PID control based on RBF neural network identification. In: 17th IEEE international conference on tools with artificial intelligence (ICTAI'05), Hong Kong, China, pp 1854-1857

61. Osman A, Alzahrani A (2019) New approach for automated epileptic disease diagnosis using an integrated self-organization map and radial basis function neural network algorithm. IEEE Access 7:4741-4747

62. Mansourkhaki A, Berangi M, Haghiri M (2018) Comparative application of radial basis function and multilayer perceptron neural networks to predict traffic noise pollution in Tehran roads. J Ecol Eng 19(1):113-121

63. Jagannath J, Furman S, Jagannath A, Ling L, Burger A, Drozd A (2019) HELPER: heterogeneous efficient low power radio for enabling ad hoc emergency public safety networks. Ad Hoc Netw 89:218-235

64. Grover J, Jain A, Chaudhari N (2019) Unmanned aerial vehicles operated emergency ad hoc networks. In: 7th International conference on communication systems and network technologies (CSNT)

65. Farhan K, Abdel-Fattah F, Altarawneh F, Lafi M (2019) Survey paper on multicast routing in mobile ad hoc networks. In: IEEE Jordan international joint conference on electrical engineering and information technology (JEEIT), Amman, Jordan

66. Demarchou E, Psomas C, Krikidis I (2019) Asynchronous ad hoc networks with wireless powered cognitive communications. IEEE Trans Cognit Commun Netw 5(2):440-451

67. Vodafone Foundation Instant Network team deployed to area devastated by Hurricane Irma. https:// www.vodafone.com/content/index/media/vodafone-group-releases/2017/instant-network-teamdeployed.html. Accessed 10 April 2019

Publisher's Note Springer Nature remains neutral with regard to jurisdictional claims in published maps and institutional affiliations. 